\title{
MEDICAL UNIVERSITY STUDENTS' PERSPECTIVE ON MARKETING OF DIETARY SUPPLEMENTS
}

\author{
ANNA PUŚCION-JAKUBIK ${ }^{1 *}$, KRZYSZTOF KUS ${ }^{2}$, and KATARZYNA SOCHA ${ }^{1}$
}

\author{
${ }^{1}$ Department of Bromatology, Faculty of Pharmacy with the Division of Laboratory Medicine, \\ Medical University of Białystok, Mickiewicza 2D, 15-222 Białystok, Poland \\ ${ }^{2}$ Department of Pharmacoeconomics and Social Pharmacy, \\ Poznan University of Medical Sciences, Rokietnicka 7, 60-806 Poznań, Poland
}

\begin{abstract}
Dietary supplements are a group of products classified as food, prepared in a pharmaceutical form like medicines. This encourages patients to be frequent and even excessive use of these preparations, including thanks to marketing activities. The aim of the study was to assess the opinions of students of the Medical University of Bialystok (MUB) on dietary supplements and their marketing. The survey was conducted using the proprietary questionnaire, among 300 students representing all faculties: Faculty of Medicine with the Division of Dentistry and Division of Medical Education In English (FM), Faculty of Pharmacy with the Division of Laboratory Medicine (FP), and Faculty of Health Sciences (FHS). It has been shown that the source of knowledge for FP students is primary classes within their studies $(80.6 \%)$. Most students of the FHS show a lack of trust in the dietary supplements of their brand pharmacy (89.2\%). Students of the MUB are a group of future specialists who, thanks to their knowledge and experience, can recommend dietary supplements that are of good quality, therefore their opinion is important.
\end{abstract}

Keywords: dietary supplements, marketing, students

Dietary supplements are foods whose purpose is to supplement the diet with deficient ingredients: minerals, vitamins, or other substances. They may have nutritional or other physiological effects, but no therapeutic effect. They appear in concentrated form, among others capsules, tablets, dragees, powder sachets, dropper bottles, liquid ampoules, etc. This form is intended to facilitate dosing (1). The regulation of the Minister of Health contains a list of 13 vitamins and 17 minerals that may be components of dietary supplements. Polish legislation, however, does not define the so-called "other substances", which allows manufacturers to market newer and newer supplements, which are a response to the created needs of consumers and patients (2).

Supplements are recommended primarily to the following groups of patients: people with vitamin and mineral deficiencies (e.g. during low-energy diets), elderly people, people excluding products or groups of products from the diet (e.g. vegetarians, lactoseintolerant patients), and pregnant women (3). At the same time, market data indicate that Poles willingly reach for dietary supplements - e.g. a study by Matysek-Nawrocka et al. (2016) indicates that $77 \%$ of respondents used dietary supplements. The remaining respondents indicated that they did not use or remember - which may indicate difficulties in distinguishing dietary supplements from drugs (4).

Dietary supplements are not as closely controlled as drugs - in the individual phase of clinical trials. This results in the fact that contaminated or adulterated dietary supplements may be on sale. For example, it was noted, contamination of dietary supplements with mercury (in tablets containing Bamboo shoots and Horsetail: $4212.04 \mu \mathrm{g} / \mathrm{kg}$, which exceeds the norm at $100 \mu \mathrm{g} / \mathrm{kg}$, which the authors refer to) (5). This fact does not discourage consumers: it is estimated that in Poland approximately $38.2 \%$ of women and $32.1 \%$ of men use dietary supplements. The most used categories include magnesium (7.56\% of the population), immunostimulants $(6.58 \%)$, and probiotics $(6.13 \%)(6)$.

Various types of marketing activities influence consumer's decisions. Their goal is to define and satisfy social needs in order to make a profit (7). One of the main places where dietary supplements are sold are pharmacies. Marketing activities undertaken by managers include: all kinds of promotional

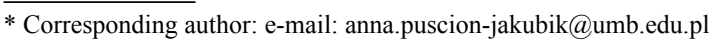


initiatives, maintaining an appropriate pricing policy, matching the range of products offered, appropriate personnel, and exposing the location of points of sale outlets. The marketing success of the pharmacy depends on the above 6 elements that make up the socalled retail mix (8). Marketing activities seem to be effective because in 2018 pharmacy and non-pharmacy sales of dietary supplements in Poland amounted to PLN 5.4 billion (9).

Therefore, the aim of the study was to assess the knowledge and opinions of students on the marketing of dietary supplements. Medical University students are a specific and important group: they are the ones who will recommend specific preparations to their patients in the future, being the reason why their opinion is extremely important. For the first time, our study presents cross-sectional data, including the views of students from all faculties of MUB.

\section{EXPERIMENTAL}

The study was carried out on 300 students of the MUB: from November 13 to December 7, 2020, using the proprietary questionnaire. The questionnaire was verified with a trial test, according to Karbownik et al. (10).

The students filled in an original questionnaire containing 31 questions, which was made available online. Questions 1 to 9 concerned the field of study and year of study, gender, age, height, body weight, subjective assessment of the material situation, health condition, and physical activity. Questions from 10 to 30 concerned the dietary supplements used and the opinions of students, and question 31 - was an open question, allowing to add own opinion. Sending the completed questionnaire was tantamount to consent to the participation in the survey. For multiple-answer questions (regarding preferences), the sum of the group sizes is greater than 300 .

Conducting an anonymous questionnaire did not require the approval of the Bioethics Committee of the Medical University of Bialystok.

In order to assess the relationship between the nominal features, the chi-square test of independence was performed. The level of statistical significance was set at $\mathrm{p}<0.05$. Microsoft Excel 2019 and Statistica 13.3 Statsoft were used for data analysis.

\section{RESULTS}

The characteristics of the surveyed group are presented in Table 1. The surveyed students ranged in age from 18.0 to 29.0 years, their BMI ranged from 15.79 to $36.78 \mathrm{~kg} / \mathrm{m}^{2}$.

Among students from the Faculty of Medicine with the Division of Dentistry and Division of Medical Education In English (FM), the largest percentage of respondents were medical students (80.3\% of respondents from this Faculty), among students from the Faculty of Pharmacy with the Division of Laboratory Medicine (FP): pharmacy students $(45.6 \%)$, and of the Faculty of Health Sciences (FHS): dietetics students: $32.4 \%$. The highest percentage of the respondents from among FM was 1st year students, among FP students: 4th year, and FHS students: 5th year (Table 2). Women constituted the greater part of the group: among students with FM, it was $80.3 \%$, with FP: $88.1 \%$, and among

Table 1. Characteristics of the studied group, taking into account the division into faculty.

\begin{tabular}{|c|c|c|c|}
\hline Faculty & $\begin{array}{l}\text { Faculty of Medicine with the } \\
\text { Division of Dentistry and Division of } \\
\text { Medical Education In English (FM) } \\
\qquad(\mathrm{n}=66)\end{array}$ & $\begin{array}{l}\text { Faculty of Pharmacy with the } \\
\text { Division of Laboratory Medicine (FP) } \\
\qquad(n=160)\end{array}$ & $\begin{array}{l}\text { Faculty of Health } \\
\text { Sciences (FHS) } \\
(\mathrm{n}=74)\end{array}$ \\
\hline \multicolumn{4}{|c|}{ Age [years] } \\
\hline $\begin{array}{c}\mathrm{Av} \pm \mathrm{SD} \\
(\mathrm{Min}-\mathrm{Max})\end{array}$ & $\begin{array}{c}22.1 \pm 2.1 \\
(19.0-27.0)\end{array}$ & $\begin{array}{c}22.4 \pm 1.9 \\
(19.0-29.0)\end{array}$ & $\begin{array}{c}21.8 \pm 1.8 \\
(18.0-26.0)\end{array}$ \\
\hline \multicolumn{4}{|c|}{ Growth $[\mathrm{cm}]$} \\
\hline $\begin{array}{c}\mathrm{Av} \pm \mathrm{SD} \\
(\mathrm{Min}-\mathrm{Max})\end{array}$ & $\begin{array}{c}169.6 \pm 7.8 \\
(152.0-186.0)\end{array}$ & $\begin{array}{c}168.5 \pm 7.1 \\
(156.0-190.0)\end{array}$ & $\begin{array}{c}167.6 \pm 8.3 \\
(151.0-195.0)\end{array}$ \\
\hline \multicolumn{4}{|c|}{ Weigh [kg] } \\
\hline $\begin{array}{c}\mathrm{Av} \pm \mathrm{SD} \\
(\mathrm{Min}-\mathrm{Max})\end{array}$ & $\begin{array}{l}62.5 \pm 11.0 \\
(46.0-96.0) \\
\end{array}$ & $\begin{array}{c}63.9 \pm 13.2 \\
(45.0-130.0) \\
\end{array}$ & $\begin{array}{l}62.3 \pm 10.8 \\
(46.0-95.0) \\
\end{array}$ \\
\hline \multicolumn{4}{|c|}{ BMI $\left[\mathrm{kg} / \mathrm{m}^{2}\right]$} \\
\hline $\begin{array}{c}\mathrm{Av} \pm \mathrm{SD} \\
(\mathrm{Min}-\mathrm{Max})\end{array}$ & $\begin{array}{c}21.67 \pm 3.01 \\
(16.26-32.08)\end{array}$ & $\begin{array}{l}22.42 \pm 3.64 \\
(16.14-36.78)\end{array}$ & $\begin{array}{l}22.06 \pm 2.48 \\
(15.79-27.78)\end{array}$ \\
\hline
\end{tabular}

Av - average, Max - maximum, Min - minimum, $\mathrm{n}$ - group size, $\mathrm{SD}$ - standard deviation 
FHS students: $86.5 \%$, which may also indirectly indicate the gender structure among students of particular fields of study. The majority of respondents assessed their physical activity as low: $69.7 \%, 75.0 \%$, and $63.5 \%$, respectively. The largest percentage of students at individual faculties were people who assessed their material situation as good $(54.5 \%$, $78.2 \%$, and $85.1 \%$ ). Similarly, the largest percentage of the respondents as those who described their health as $\operatorname{good}(74.2 \%, 63.8 \%$, and $70.3 \%)$ (Table 2).

Table 2. Data on the field and year of study of the respondents, their gender, physical activity, and subjective assessment of the material situation and health.

\begin{tabular}{|c|c|c|c|}
\hline Faculty & $\begin{array}{l}\text { Faculty of Medicine with the } \\
\text { Division of Dentistry and } \\
\text { Division of Medical Education } \\
\text { In English (FM) } \\
(\mathrm{n}=66)\end{array}$ & $\begin{array}{l}\text { Faculty of Pharmacy with } \\
\text { the Division of Laboratory } \\
\text { Medicine (FP) } \\
(n=160)\end{array}$ & $\begin{array}{l}\text { Faculty of Health } \\
\text { Sciences (FHS) } \\
\quad(\mathrm{n}=74)\end{array}$ \\
\hline \multicolumn{4}{|c|}{ Courses of study } \\
\hline \multirow{8}{*}{ Courses } & \multirow{3}{*}{$\begin{array}{c}\text { Medicine: } \\
80.3 \%(\mathrm{n}=53)\end{array}$} & \multirow{2}{*}{$\begin{array}{c}\text { Pharmacy: } \\
45.6 \%(\mathrm{n}=73)\end{array}$} & $\begin{array}{c}\text { Dietetics: } \\
32.4 \%(\mathrm{n}=24)\end{array}$ \\
\hline & & & $\begin{array}{l}\text { Physiotherapy: } \\
20.3 \%(n=15)\end{array}$ \\
\hline & & \multirow{2}{*}{$\begin{array}{l}\text { Cosmetology: } \\
38.1 \%(\mathrm{n}=61)\end{array}$} & $\begin{array}{c}\text { Nursing: } \\
17.6 \%(\mathrm{n}=13)\end{array}$ \\
\hline & \multirow{3}{*}{$\begin{array}{c}\text { Dentistry: } \\
18.2 \%(\mathrm{n}=12)\end{array}$} & & $\begin{array}{c}\text { Emergency medicine: } \\
6.8 \%(\mathrm{n}=5)\end{array}$ \\
\hline & & \multirow{2}{*}{$\begin{array}{l}\text { Laboratory medicine: } \\
12.5 \%(\mathrm{n}=20)\end{array}$} & $\begin{array}{l}\text { Midwifery: } \\
6.8 \%(\mathrm{n}=5)\end{array}$ \\
\hline & & & $\begin{array}{l}\text { Speach therapy and } \\
\text { phonoaudiology: } \\
6.8 \%(\mathrm{n}=5)\end{array}$ \\
\hline & \multirow{2}{*}{$\begin{array}{c}\text { Dental technology: } \\
1.5 \%(\mathrm{n}=1)\end{array}$} & \multirow{2}{*}{$\begin{array}{l}\text { PhD studies: } \\
3.8 \%(\mathrm{n}=6)\end{array}$} & $\begin{array}{l}\text { Electroradiology: } \\
5.4 \%(\mathrm{n}=4)\end{array}$ \\
\hline & & & $\begin{array}{l}\text { Public health: } \\
3.9 \%(\mathrm{n}=3)\end{array}$ \\
\hline \multicolumn{4}{|c|}{ Year of study } \\
\hline $\mathrm{I}$ & $22.7 \%(n=15)$ & $10.6 \%(\mathrm{n}=17)$ & $17.5 \%(n=13)$ \\
\hline II & $13.6 \%(\mathrm{n}=9)$ & $16.3 \%(\mathrm{n}=26)$ & $16.2 \%(n=12)$ \\
\hline III & $15.3 \%(n=10)$ & $6.3 \%(n=10)$ & $12.2 \%(\mathrm{n}=9)$ \\
\hline IV & $13.6 \%(n=9)$ & $29.4 \%(n=47)$ & $18.9 \%(n=14)$ \\
\hline $\mathrm{V}$ & $19.7 \%(\mathrm{n}=13)$ & $26.2 \%(n=42)$ & $33.8 \%(n=25)$ \\
\hline VI & $12.1 \%(\mathrm{n}=8)$ & $7.5 \%(n=12)$ & $0.0 \%(\mathrm{n}=0)$ \\
\hline Graduate & $1.5 \%(\mathrm{n}=1)$ & $0.0 \%(\mathrm{n}=0)$ & $0.0 \%(\mathrm{n}=0)$ \\
\hline Intern & $1.5 \%(n=1)$ & $0.0 \%(\mathrm{n}=0)$ & $1.4 \%(n=1)$ \\
\hline $\mathrm{PhD}$ & $0.0 \%(\mathrm{n}=0)$ & $3.7 \%(n=6)$ & $0.0 \%(\mathrm{n}=0)$ \\
\hline \multicolumn{4}{|c|}{ Gender } \\
\hline Woman & $80.3 \%(n=53)$ & $88.1 \%(\mathrm{n}=141)$ & $86.5 \%(n=64)$ \\
\hline Man & $19.7 \%(n=13)$ & $11.9 \%(n=19)$ & $13.5 \%(n=10)$ \\
\hline \multicolumn{4}{|c|}{ Physical activity } \\
\hline $\begin{array}{c}\text { Low (occasional exercise } \\
1-3 \text { times a week) }\end{array}$ & $69.7 \%(n=46)$ & $75.0 \%(n=120)$ & $63.5 \%(n=47)$ \\
\hline $\begin{array}{c}\text { Moderate } \\
\text { (1 hour of activity per day) }\end{array}$ & $24.2 \%(\mathrm{n}=16)$ & $21.9 \%(n=35)$ & $35.1 \%(n=26)$ \\
\hline $\begin{array}{c}\text { High } \\
\text { (heavy physical flare and } \\
\text { daily workouts) }\end{array}$ & $6.1 \%(n=4)$ & $3.1 \%(\mathrm{n}=5)$ & $1.4 \%(n=1)$ \\
\hline
\end{tabular}


Table 2. Data on the field and year of study of the respondents, their gender, physical activity, and subjective assessment of the material situation and health (cont.).

\begin{tabular}{|c|c|c|c|}
\hline Faculty & $\begin{array}{l}\text { Faculty of Medicine with the } \\
\text { Division of Dentistry and } \\
\text { Division of Medical Education } \\
\text { In English (FM) } \\
(\mathrm{n}=66)\end{array}$ & $\begin{array}{l}\text { Faculty of Pharmacy with } \\
\text { the Division of Laboratory } \\
\text { Medicine (FP) } \\
(n=160)\end{array}$ & $\begin{array}{l}\text { Faculty of Health } \\
\text { Sciences (FHS) } \\
(n=74)\end{array}$ \\
\hline \multicolumn{4}{|c|}{ Subjective assessment of the material status } \\
\hline Very good & $40.9 \%(n=27)$ & $16.8 \%(n=27)$ & $9.5 \%(n=7)$ \\
\hline Good & $54.5 \%(\mathrm{n}=36)$ & $78.2 \%(\mathrm{n}=125)$ & $85.1 \%(n=63)$ \\
\hline $\mathrm{Bad}$ & $4.6 \%(n=3)$ & $5.0 \%(\mathrm{n}=8)$ & $5.4 \%(n=4)$ \\
\hline \multicolumn{4}{|c|}{ Subjective health assessment } \\
\hline Very good & $21.2 \%(n=14)$ & $31.3 \%(n=50)$ & $23.0 \%(\mathrm{n}=17)$ \\
\hline Good & $74.2 \%(n=49)$ & $63.8 \%(n=102)$ & $70.3 \%(n=52)$ \\
\hline Bad & $4.6 \%(n=3)$ & $4.9 \%(n=8)$ & $6.7 \%(n=5)$ \\
\hline
\end{tabular}

$\mathrm{n}$ - group size

Table 3 presents data on the opinions and experiences of students with regard to dietary supplements. Three divisions of the studied group were taken into account: in terms of faculty, gender, and period of study. Statistical analyzes were performed within the above groups.
Opinions are divided as to what a dietary supplement is: about three-quarters of students stated that it is a food aimed at supplementing nutritional deficiencies (FM: 77.3\%, FP: 77.5\%, FHS: 70.3\%). Significantly more men answered this question correctly $(85.7 \%)$.

Table 3. Dietary supplements in the opinion of students of three faculties of the Medical University in Białystok.

\begin{tabular}{|c|c|c|c|c|c|c|c|}
\hline Division & \multicolumn{3}{|c|}{ Faculty } & \multicolumn{2}{|c|}{ Gender } & \multicolumn{2}{|c|}{ Period of study } \\
\hline Parameters & $\begin{array}{l}\% \text { of FM } \\
\text { students } \\
(n=66)\end{array}$ & $\begin{array}{l}\% \text { of FP } \\
\text { students } \\
(n=160)\end{array}$ & $\begin{array}{c}\% \text { of FHS } \\
\text { students } \\
(\mathrm{n}=74)\end{array}$ & $\begin{array}{c}\% \text { of } \\
\text { women } \\
(\mathrm{W}) \\
(\mathrm{n}=258)\end{array}$ & $\begin{array}{c}\% \text { of men } \\
(\mathrm{M}) \\
(\mathrm{n}=42)\end{array}$ & $\begin{array}{c}\% \text { of } \\
\text { students on } \\
\text { I-III years } \\
(\mathrm{I} \text { group) } \\
(\mathrm{n}=121)\end{array}$ & $\begin{array}{l}\% \text { of students } \\
\text { of subsequent } \\
\text { years of study } \\
\text { (II group) } \\
(\mathrm{n}=179)\end{array}$ \\
\hline \multicolumn{8}{|c|}{ Question: What is a dietary supplement? } \\
\hline $\begin{array}{l}\text { A drug, a preparation } \\
\text { that treats nutritional } \\
\text { deficiencies }\end{array}$ & $\begin{array}{l}22.7 \% \\
(\mathrm{n}=15)\end{array}$ & $\begin{array}{l}22.5 \% \\
(n=36)\end{array}$ & $\begin{array}{l}29.7 \% \\
(n=22)\end{array}$ & $\begin{array}{l}26.0 \% \\
(n=67)\end{array}$ & $\begin{array}{l}14.3 \% \\
(n=6)\end{array}$ & $\begin{array}{l}24.8 \% \\
(n=30)\end{array}$ & $\begin{array}{l}24.0 \% \\
(\mathrm{n}=43)\end{array}$ \\
\hline $\begin{array}{l}\text { It is food, it supplements } \\
\text { nutritional deficiencies }\end{array}$ & $\begin{array}{l}77.3 \% \\
(n=51)\end{array}$ & $\begin{array}{c}77.5 \% \\
(n=124)\end{array}$ & $\begin{array}{l}70.3 \% \\
(n=52)\end{array}$ & $\begin{array}{c}74.0 \% \\
(n=191)\end{array}$ & $\begin{array}{c}85.7 \% \\
(\mathrm{n}=36)^{*}\end{array}$ & $\begin{array}{l}75.2 \% \\
(n=91)\end{array}$ & $\begin{array}{c}76.0 \% \\
(n=136)\end{array}$ \\
\hline \multicolumn{8}{|c|}{ Question: What do you primarily follow when purchasing a dietary supplement? } \\
\hline Price & $\begin{array}{l}40.9 \% \\
(n=27)\end{array}$ & $\begin{array}{l}37.5 \% \\
(n=60)\end{array}$ & $\begin{array}{l}43.2 \% \\
(n=32)\end{array}$ & $\begin{array}{c}39.9 \% \\
(\mathrm{n}=103)\end{array}$ & $\begin{array}{l}38.1 \% \\
(\mathrm{n}=16)\end{array}$ & $\begin{array}{l}42.1 \% \\
(n=51)\end{array}$ & $\begin{array}{l}38.0 \% \\
(n=68)\end{array}$ \\
\hline $\begin{array}{l}\text { Composition (chemical } \\
\text { form) }\end{array}$ & $\begin{array}{l}77.3 \% \\
(n=51)\end{array}$ & $\begin{array}{c}90.0 \% \\
(\mathrm{n}=128)^{*}\end{array}$ & $\begin{array}{l}71.6 \% \\
(n=53)\end{array}$ & $\begin{array}{c}77.5 \% \\
(n=200)\end{array}$ & $\begin{array}{l}76.2 \% \\
(n=32)\end{array}$ & $\begin{array}{l}69.4 \% \\
(n=84)\end{array}$ & $\begin{array}{c}82.7 \% \\
(\mathrm{n}=148)\end{array}$ \\
\hline Habit & $\begin{array}{l}7.6 \% \\
(n=5)\end{array}$ & $\begin{array}{c}7.5 \% \\
(n=12)\end{array}$ & $\begin{array}{l}8.1 \% \\
(n=6)\end{array}$ & $\begin{array}{c}7.8 \% \\
(n=20)\end{array}$ & $\begin{array}{c}7.1 \% \\
(n=3)\end{array}$ & $\begin{array}{c}8.3 \% \\
(n=10)\end{array}$ & $\begin{array}{c}7.3 \% \\
(n=13)\end{array}$ \\
\hline Advertisement & $\begin{array}{c}0.0 \% \\
(\mathrm{n}=0)\end{array}$ & $2.5 \%(n=4)$ & $\begin{array}{c}6.8 \% \\
(\mathrm{n}=5)\end{array}$ & $\begin{array}{c}3.5 \% \\
(n=9)\end{array}$ & $\begin{array}{c}0.0 \% \\
(\mathrm{n}=0)\end{array}$ & $\begin{array}{c}3.3 \% \\
(n=4)\end{array}$ & $\begin{array}{l}2.8 \% \\
(n=5)\end{array}$ \\
\hline $\begin{array}{l}\text { Pharmacist's } \\
\text { recommendation }\end{array}$ & $\begin{array}{c}39.4 \% \\
(\mathrm{n}=26)\end{array}$ & $\begin{array}{l}43.8 \% \\
(n=70)\end{array}$ & $\begin{array}{l}24.3 \% \\
(\mathrm{n}=18)\end{array}$ & $\begin{array}{c}40.3 \% \\
(\mathrm{n}=104)^{*}\end{array}$ & $\begin{array}{l}23.9 \% \\
(\mathrm{n}=10)\end{array}$ & $\begin{array}{c}42.1 \% \\
(n=51)\end{array}$ & $\begin{array}{l}35.2 \% \\
(\mathrm{n}=63)\end{array}$ \\
\hline Brand/company & $\begin{array}{c}3.0 \% \\
(\mathrm{n}=2)\end{array}$ & $\begin{array}{c}16.9 \% \\
(n=27)\end{array}$ & $\begin{array}{c}8.1 \% \\
(n=6)\end{array}$ & $\begin{array}{l}11.2 \% \\
(n=29)\end{array}$ & $\begin{array}{l}14.3 \% \\
(n=6)\end{array}$ & $\begin{array}{c}5.0 \% \\
(\mathrm{n}=6)\end{array}$ & $\begin{array}{c}16.2 \% \\
(n=29)\end{array}$ \\
\hline Doctor's recommendation & $\begin{array}{c}39.4 \% \\
(n=26)\end{array}$ & $\begin{array}{c}20.6 \% \\
(n=33)\end{array}$ & $\begin{array}{c}31.1 \% \\
(n=23)\end{array}$ & $\begin{array}{c}29.5 \% \\
(n=76)\end{array}$ & $\begin{array}{l}14.3 \% \\
(n=6)\end{array}$ & $\begin{array}{c}28.1 \% \\
(n=34)\end{array}$ & $\begin{array}{c}26.8 \% \\
(n=48)\end{array}$ \\
\hline
\end{tabular}


Table 3. Dietary supplements in the opinion of students of three faculties of the Medical University in Białystok (cont.).

\begin{tabular}{|c|c|c|c|c|c|c|c|}
\hline Division & \multicolumn{3}{|c|}{ Faculty } & \multicolumn{2}{|c|}{ Gender } & \multicolumn{2}{|c|}{ Period of study } \\
\hline Parameters & $\begin{array}{c}\% \text { of FM } \\
\text { students } \\
(n=66)\end{array}$ & $\begin{array}{l}\% \text { of FP } \\
\text { students } \\
(\mathrm{n}=160)\end{array}$ & $\begin{array}{c}\% \text { of FHS } \\
\text { students } \\
(\mathrm{n}=74)\end{array}$ & $\begin{array}{c}\% \text { of } \\
\text { women } \\
(\mathrm{W}) \\
(\mathrm{n}=258)\end{array}$ & $\begin{array}{c}\% \text { of men } \\
(\mathrm{M}) \\
(\mathrm{n}=42)\end{array}$ & \begin{tabular}{|c}
$\quad \%$ of \\
students on \\
I-III years \\
(I group) \\
$(\mathrm{n}=121)$
\end{tabular} & $\begin{array}{c}\% \text { of students } \\
\text { of subsequent } \\
\text { years of study } \\
\text { (II group) } \\
(\mathrm{n}=179)\end{array}$ \\
\hline Packaging & $\begin{array}{c}1.5 \% \\
(\mathrm{n}=1)\end{array}$ & $\begin{array}{c}1.3 \% \\
(\mathrm{n}=2)\end{array}$ & $\begin{array}{c}2.7 \% \\
(\mathrm{n}=2)\end{array}$ & $\begin{array}{l}1.6 \% \\
(\mathrm{n}=4)\end{array}$ & $\begin{array}{c}2.4 \% \\
(\mathrm{n}=1)\end{array}$ & $\begin{array}{l}2.5 \% \\
(\mathrm{n}=3)\end{array}$ & $\begin{array}{c}1.1 \% \\
(\mathrm{n}=2)\end{array}$ \\
\hline $\begin{array}{c}\text { Another reason - total: } \\
\text { - the highest price, } \\
\text { - recommendation by } \\
\text { someone (e.g. on a blog), } \\
\text { - dose, } \\
\text { - recommendation of } \\
\text { a dietitian, } \\
\text { - drug status, } \\
\text { not supplement status, } \\
\text { - quality tests, } \\
\text { - needs and knowledge, } \\
\text { - place of purchase: } \\
\text { pharmacy, } \\
\text { - lecturer's } \\
\text { recommendation }\end{array}$ & $\begin{array}{c}3.0 \% \\
(\mathrm{n}=2)\end{array}$ & $\begin{array}{c}5.0 \% \\
(\mathrm{n}=8)\end{array}$ & $\begin{array}{c}4.2 \% \\
(n=3)\end{array}$ & $\begin{array}{c}4.0 \% \\
(\mathrm{n}=10)\end{array}$ & $\begin{array}{c}7.2 \% \\
(\mathrm{n}=3)\end{array}$ & $\begin{array}{c}3.2 \% \\
(\mathrm{n}=5)\end{array}$ & $\begin{array}{c}4.6 \% \\
(n=8)\end{array}$ \\
\hline Does not take supplements & $\begin{array}{c}6.0 \% \\
(\mathrm{n}=4)\end{array}$ & $\begin{array}{l}1.9 \% \\
(\mathrm{n}=3)\end{array}$ & $\begin{array}{c}1.4 \% \\
(\mathrm{n}=1)\end{array}$ & $\begin{array}{c}1.6 \% \\
(n=4)\end{array}$ & $\begin{array}{l}9.5 \% \\
(n=4)\end{array}$ & $\begin{array}{c}2.5 \% \\
(\mathrm{n}=3)\end{array}$ & $\begin{array}{c}2.8 \% \\
(\mathrm{n}=5)\end{array}$ \\
\hline \multicolumn{8}{|c|}{ Question: The main sources of knowledge about dietary supplements } \\
\hline Internet & $\begin{array}{c}66.7 \% \\
(\mathrm{n}=44)\end{array}$ & $\begin{array}{c}65.6 \% \\
(\mathrm{n}=105)\end{array}$ & $\begin{array}{c}73.0 \% \\
(\mathrm{n}=54)\end{array}$ & $\begin{array}{c}65.5 \% \\
(\mathrm{n}=169)\end{array}$ & $\begin{array}{c}81.0 \% \\
(\mathrm{n}=34)\end{array}$ & $\begin{array}{c}65.3 \% \\
(\mathrm{n}=79)\end{array}$ & $\begin{array}{c}69.3 \% \\
(\mathrm{n}=124)\end{array}$ \\
\hline TV commercials & $\begin{array}{c}9.1 \% \\
(\mathrm{n}=6)\end{array}$ & $\begin{array}{c}8.1 \% \\
(\mathrm{n}=13)\end{array}$ & $\begin{array}{c}8.1 \% \\
(\mathrm{n}=6)\end{array}$ & $\begin{array}{c}8.5 \% \\
(\mathrm{n}=22)\end{array}$ & $\begin{array}{c}7.1 \% \\
(\mathrm{n}=3)\end{array}$ & $\begin{array}{c}12.4 \% \\
(\mathrm{n}=15)\end{array}$ & $\begin{array}{c}5.6 \% \\
(\mathrm{n}=10)\end{array}$ \\
\hline Professional press & $\begin{array}{c}24.2 \% \\
(\mathrm{n}=16)\end{array}$ & $\begin{array}{c}18.1 \% \\
(n=29)\end{array}$ & $\begin{array}{c}17.6 \% \\
(n=13)\end{array}$ & $\begin{array}{c}19.0 \% \\
(\mathrm{n}=49)\end{array}$ & $\begin{array}{l}21.4 \% \\
(\mathrm{n}=9)\end{array}$ & $\begin{array}{c}14.0 \% \\
(\mathrm{n}=17)\end{array}$ & $\begin{array}{c}22.9 \% \\
(\mathrm{n}=41)\end{array}$ \\
\hline Friends and family & $\begin{array}{c}25.8 \% \\
(\mathrm{n}=17)\end{array}$ & $\begin{array}{c}14.4 \% \\
(n=23)\end{array}$ & $\begin{array}{c}14.9 \% \\
(\mathrm{n}=11)\end{array}$ & $\begin{array}{c}17.8 \% \\
(n=46)\end{array}$ & $\begin{array}{l}11.9 \% \\
(\mathrm{n}=5)\end{array}$ & $\begin{array}{c}25.6 \% \\
(\mathrm{n}=31)\end{array}$ & $\begin{array}{c}11.2 \% \\
(\mathrm{n}=20)\end{array}$ \\
\hline Studies & $\begin{array}{c}57.6 \% \\
(\mathrm{n}=38)\end{array}$ & $\begin{array}{c}80.6 \% \\
(\mathrm{n}=129)^{* *}\end{array}$ & $\begin{array}{c}43.2 \% \\
(n=32)\end{array}$ & $\begin{array}{c}66.7 \% \\
(\mathrm{n}=172)\end{array}$ & $\begin{array}{c}64.3 \% \\
(\mathrm{n}=27)\end{array}$ & $\begin{array}{c}51.2 \% \\
(\mathrm{n}=62)\end{array}$ & $\begin{array}{c}76.5 \% \\
(\mathrm{n}=137)^{* *}\end{array}$ \\
\hline Pharmacist & $\begin{array}{c}37.9 \% \\
(\mathrm{n}=25)\end{array}$ & $\begin{array}{c}41.3 \% \\
(n=66)\end{array}$ & $\begin{array}{c}20.3 \% \\
(\mathrm{n}=15)\end{array}$ & $\begin{array}{c}37.2 \% \\
(\mathrm{n}=96)\end{array}$ & $\begin{array}{c}23.8 \% \\
(\mathrm{n}=10)\end{array}$ & $\begin{array}{c}43.0 \% \\
(n=52)\end{array}$ & $\begin{array}{c}30.2 \% \\
(\mathrm{n}=54)\end{array}$ \\
\hline Doctor & $\begin{array}{c}42.4 \% \\
(n=28)\end{array}$ & $\begin{array}{c}19.4 \% \\
(\mathrm{n}=31)\end{array}$ & $\begin{array}{c}18.9 \% \\
(n=14)\end{array}$ & $\begin{array}{c}26.0 \% \\
(\mathrm{n}=67)\end{array}$ & $\begin{array}{l}14.3 \% \\
(\mathrm{n}=6)\end{array}$ & $\begin{array}{c}31.4 \% \\
(\mathrm{n}=38)\end{array}$ & $\begin{array}{c}19.6 \% \\
(\mathrm{n}=35)\end{array}$ \\
\hline \multicolumn{8}{|c|}{ Question: Have you noticed a beneficial effect of using supplements? } \\
\hline Yes & $\begin{array}{c}56.1 \% \\
(\mathrm{n}=37)\end{array}$ & $\begin{array}{c}54.4 \% \\
(\mathrm{n}=87)\end{array}$ & $\begin{array}{c}55.4 \% \\
(\mathrm{n}=41)\end{array}$ & $\begin{array}{c}56.9 \% \\
(\mathrm{n}=146)\end{array}$ & $\begin{array}{c}45.2 \% \\
(n=19)\end{array}$ & $\begin{array}{c}54.5 \% \\
(\mathrm{n}=66)\end{array}$ & $\begin{array}{c}55.3 \% \\
(\mathrm{n}=99)\end{array}$ \\
\hline No & $\begin{array}{c}25.8 \% \\
(\mathrm{n}=17)\end{array}$ & $\begin{array}{c}16.2 \% \\
(\mathrm{n}=26)\end{array}$ & $\begin{array}{c}25.7 \% \\
(\mathrm{n}=19)\end{array}$ & $\begin{array}{c}20.2 \% \\
(\mathrm{n}=52)\end{array}$ & $\begin{array}{c}23.8 \% \\
(\mathrm{n}=10)\end{array}$ & $\begin{array}{c}22.3 \% \\
(\mathrm{n}=27)\end{array}$ & $\begin{array}{c}19.6 \% \\
(\mathrm{n}=35)\end{array}$ \\
\hline Not applicable & $\begin{array}{c}18.1 \% \\
(\mathrm{n}=12)\end{array}$ & $\begin{array}{c}29.4 \% \\
(\mathrm{n}=47)\end{array}$ & $\begin{array}{c}18.9 \% \\
(\mathrm{n}=14)\end{array}$ & $\begin{array}{c}22.9 \% \\
(\mathrm{n}=60)\end{array}$ & $\begin{array}{c}31.0 \% \\
(\mathrm{n}=13)\end{array}$ & $\begin{array}{c}23.2 \% \\
(\mathrm{n}=28)\end{array}$ & $\begin{array}{c}25.1 \% \\
(\mathrm{n}=45)\end{array}$ \\
\hline \multicolumn{8}{|c|}{ Question: Have you noticed any side effects of dietary supplements? } \\
\hline Yes & $\begin{array}{c}4.5 \% \\
(\mathrm{n}=3)\end{array}$ & $\begin{array}{c}3.7 \% \\
(\mathrm{n}=6)\end{array}$ & $\begin{array}{c}2.7 \% \\
(\mathrm{n}=2)\end{array}$ & $\begin{array}{c}3.5 \% \\
(\mathrm{n}=9)\end{array}$ & $\begin{array}{c}4.8 \% \\
(n=2)\end{array}$ & $\begin{array}{c}1.7 \% \\
(\mathrm{n}=2)\end{array}$ & $\begin{array}{c}5.0 \% \\
(\mathrm{n}=9)\end{array}$ \\
\hline No & $\begin{array}{c}78.8 \% \\
(\mathrm{n}=52)\end{array}$ & $\begin{array}{c}70.0 \% \\
(\mathrm{n}=112)\end{array}$ & $\begin{array}{c}78.4 \% \\
(\mathrm{n}=58)\end{array}$ & $\begin{array}{c}75.6 \% \\
(\mathrm{n}=195)\end{array}$ & $\begin{array}{c}64.3 \% \\
(\mathrm{n}=27)\end{array}$ & $\begin{array}{c}78.5 \% \\
(\mathrm{n}=95)\end{array}$ & $\begin{array}{c}70.9 \% \\
(\mathrm{n}=127)\end{array}$ \\
\hline Not applicable & $\begin{array}{c}16.7 \% \\
(\mathrm{n}=11)\end{array}$ & $\begin{array}{l}26.3 \% \\
(\mathrm{n}=42)\end{array}$ & $\begin{array}{c}18.9 \% \\
(\mathrm{n}=14)\end{array}$ & $\begin{array}{c}20.9 \% \\
(\mathrm{n}=54)\end{array}$ & $\begin{array}{c}30.9 \% \\
(\mathrm{n}=13)\end{array}$ & $\begin{array}{c}19.8 \% \\
(n=24)\end{array}$ & $\begin{array}{c}24.1 \% \\
(\mathrm{n}=43)\end{array}$ \\
\hline
\end{tabular}


Table 3. Dietary supplements in the opinion of students of three faculties of the Medical University in Białystok (cont.).

\begin{tabular}{|c|c|c|c|c|c|c|c|}
\hline Division & \multicolumn{3}{|c|}{ Faculty } & \multicolumn{2}{|c|}{ Gender } & \multicolumn{2}{|c|}{ Period of study } \\
\hline Parameters & $\begin{array}{c}\% \text { of FM } \\
\text { students } \\
(n=66)\end{array}$ & $\begin{array}{l}\% \text { of FP } \\
\text { students } \\
(\mathrm{n}=160)\end{array}$ & $\begin{array}{c}\% \text { of FHS } \\
\text { students } \\
(\mathrm{n}=74)\end{array}$ & $\begin{array}{c}\% \text { of } \\
\text { women } \\
(\mathrm{W}) \\
(\mathrm{n}=258)\end{array}$ & $\begin{array}{c}\% \text { of men } \\
(\mathrm{M}) \\
(\mathrm{n}=42)\end{array}$ & $\begin{array}{c}\% \text { of } \\
\text { students on } \\
\text { I-III years } \\
\text { (I group) } \\
(\mathrm{n}=121)\end{array}$ & $\begin{array}{l}\% \text { of students } \\
\text { of subsequent } \\
\text { years of study } \\
\text { (II group) } \\
(\mathrm{n}=179)\end{array}$ \\
\hline \multicolumn{8}{|c|}{ Question: Do you regularly use dietary supplements? } \\
\hline Yes & $\begin{array}{c}56.1 \% \\
(n=37)\end{array}$ & $\begin{array}{c}39.4 \% \\
(n=63)\end{array}$ & $\begin{array}{c}51.4 \% \\
(n=38)\end{array}$ & $\begin{array}{c}47.3 \% \\
(n=122)\end{array}$ & $\begin{array}{c}38.1 \% \\
(n=16)\end{array}$ & $\begin{array}{c}48.8 \% \\
(n=59)\end{array}$ & $\begin{array}{c}44.1 \% \\
(n=79)\end{array}$ \\
\hline No & $\begin{array}{c}36.4 \% \\
(n=24)\end{array}$ & $\begin{array}{c}43.7 \% \\
(n=70)\end{array}$ & $\begin{array}{c}33.8 \% \\
(n=25)\end{array}$ & $\begin{array}{c}39.5 \% \\
(n=102)\end{array}$ & $\begin{array}{c}40.5 \% \\
(n=17)\end{array}$ & $\begin{array}{c}36.4 \% \\
(n=44)\end{array}$ & $\begin{array}{c}41.9 \% \\
(n=75)\end{array}$ \\
\hline Not applicable & $\begin{array}{c}7.5 \% \\
(n=5)\end{array}$ & $\begin{array}{c}16.9 \% \\
(n=27)\end{array}$ & $\begin{array}{c}14.8 \% \\
(n=11)\end{array}$ & $\begin{array}{c}13.2 \% \\
(n=34)\end{array}$ & $\begin{array}{l}21.4 \% \\
(n=9)\end{array}$ & $\begin{array}{c}14.8 \% \\
(n=18)\end{array}$ & $\begin{array}{c}14.0 \% \\
(n=25)\end{array}$ \\
\hline \multicolumn{8}{|c|}{ Question: How much PLN per month do you spend on dietary supplements? } \\
\hline Less than PLN 10 & $\begin{array}{c}6.1 \% \\
(n=4)\end{array}$ & $\begin{array}{c}2.5 \% \\
(n=4)\end{array}$ & $\begin{array}{c}14.7 \% \\
(\mathrm{n}=11)\end{array}$ & $\begin{array}{c}2.7 \% \\
(\mathrm{n}=7)\end{array}$ & $\begin{array}{c}28.6 \% \\
(n=12)\end{array}$ & $\begin{array}{c}5.0 \% \\
(n=6)\end{array}$ & $\begin{array}{c}7.3 \% \\
(n=13)\end{array}$ \\
\hline $10-19$ & $\begin{array}{c}30.3 \% \\
(n=20)\end{array}$ & $\begin{array}{c}11.9 \% \\
(n=19)\end{array}$ & $\begin{array}{c}0.0 \% \\
(n=0)\end{array}$ & $\begin{array}{c}13.5 \% \\
(n=35)\end{array}$ & $\begin{array}{c}9.5 \% \\
(n=4)\end{array}$ & $\begin{array}{c}17.4 \% \\
(n=21)\end{array}$ & $\begin{array}{c}10.1 \% \\
(n=18)\end{array}$ \\
\hline $20-40$ & $\begin{array}{c}27.3 \% \\
(n=18)\end{array}$ & $\begin{array}{c}32.5 \% \\
(n=52)\end{array}$ & $\begin{array}{c}35.1 \% \\
(n=26)\end{array}$ & $\begin{array}{c}37.2 \% \\
(n=96)\end{array}$ & $\begin{array}{c}0.0 \% \\
(\mathrm{n}=0)\end{array}$ & $\begin{array}{c}35.5 \% \\
(n=43)\end{array}$ & $\begin{array}{c}29.6 \% \\
(n=53)\end{array}$ \\
\hline $41-100$ & $\begin{array}{c}3.0 \% \\
(n=2)\end{array}$ & $\begin{array}{c}11.3 \% \\
(\mathrm{n}=18)\end{array}$ & $\begin{array}{l}12.2 \% \\
(n=9)\end{array}$ & $\begin{array}{c}10.5 \% \\
(n=27)\end{array}$ & $\begin{array}{c}4.8 \% \\
(n=2)\end{array}$ & $\begin{array}{c}7.4 \% \\
(n=9)\end{array}$ & $\begin{array}{c}11.2 \% \\
(n=20)\end{array}$ \\
\hline above 100 & $\begin{array}{c}3.0 \% \\
(n=2)\end{array}$ & $\begin{array}{c}0.6 \% \\
(n=1)\end{array}$ & $\begin{array}{c}2.7 \% \\
(n=2)\end{array}$ & $\begin{array}{c}1.6 \% \\
(n=4)\end{array}$ & $\begin{array}{c}2.4 \% \\
(\mathrm{n}=1)\end{array}$ & $\begin{array}{c}0.8 \% \\
(n=1)\end{array}$ & $\begin{array}{c}2.2 \% \\
(n=4)\end{array}$ \\
\hline I do not know & $\begin{array}{c}4.5 \% \\
(n=3)\end{array}$ & $\begin{array}{c}0.6 \% \\
(n=1)\end{array}$ & $\begin{array}{c}2.7 \% \\
(n=2)\end{array}$ & $\begin{array}{c}1.2 \% \\
(n=3)\end{array}$ & $\begin{array}{c}7.1 \% \\
(n=3)\end{array}$ & $\begin{array}{c}2.5 \% \\
(n=3)\end{array}$ & $\begin{array}{c}1.6 \% \\
(n=3)\end{array}$ \\
\hline Not applicable & $\begin{array}{c}25.8 \% \\
(n=17)\end{array}$ & $\begin{array}{c}40.6 \% \\
(\mathrm{n}=65)^{* * *}\end{array}$ & $\begin{array}{c}32.6 \% \\
(n=24)\end{array}$ & $\begin{array}{l}33.3 \% \\
(n=86)\end{array}$ & $\begin{array}{c}47.6 \% \\
(n=20)\end{array}$ & $\begin{array}{c}31.4 \% \\
(\mathrm{n}=38)\end{array}$ & $\begin{array}{l}38.0 \% \\
(n=68)\end{array}$ \\
\hline \multicolumn{8}{|c|}{ Question: Where do you buy dietary supplements? } \\
\hline At the pharmacy & $\begin{array}{l}80.3 \% \\
(n=53)\end{array}$ & $\begin{array}{c}86.9 \% \\
(\mathrm{n}=139)\end{array}$ & $\begin{array}{l}87.8 \% \\
(n=65)\end{array}$ & $\begin{array}{c}88.0 \% \\
(\mathrm{n}=227)\end{array}$ & $\begin{array}{l}71.4 \% \\
(n=30)\end{array}$ & $\begin{array}{c}87.6 \% \\
(n=106)\end{array}$ & $\begin{array}{c}84.4 \% \\
(n=151)\end{array}$ \\
\hline In a store / cosmetic store & $\begin{array}{l}10.6 \% \\
(n=7)\end{array}$ & $\begin{array}{c}5.0 \% \\
(n=8)\end{array}$ & $\begin{array}{l}9.5 \% \\
(n=7)\end{array}$ & $\begin{array}{c}7.0 \% \\
(n=18)\end{array}$ & $\begin{array}{l}9.5 \% \\
(n=4)\end{array}$ & $\begin{array}{c}6.6 \% \\
(n=8)\end{array}$ & $\begin{array}{c}7.8 \% \\
(n=14)\end{array}$ \\
\hline $\begin{array}{l}\text { In the herbal and medical } \\
\text { store }\end{array}$ & $\begin{array}{l}3.0 \% \\
(n=2)\end{array}$ & $\begin{array}{c}7.5 \% \\
(n=12)\end{array}$ & $\begin{array}{l}5.4 \% \\
(n=4)\end{array}$ & $\begin{array}{c}6.9 \% \\
(n=17)\end{array}$ & $\begin{array}{c}2.4 \% \\
(\mathrm{n}=1)\end{array}$ & $\begin{array}{c}7.4 \% \\
(n=9)\end{array}$ & $\begin{array}{l}5.0 \% \\
(n=9)\end{array}$ \\
\hline Over the Internet & $\begin{array}{l}19.7 \% \\
(n=13)\end{array}$ & $\begin{array}{l}25.6 \% \\
(n=41)\end{array}$ & $\begin{array}{c}31.1 \% \\
(n=23)\end{array}$ & $\begin{array}{l}25.6 \% \\
(n=66)\end{array}$ & $\begin{array}{l}26.2 \% \\
(\mathrm{n}=11)\end{array}$ & $\begin{array}{l}19.8 \% \\
(n=24)\end{array}$ & $\begin{array}{l}29.6 \% \\
(\mathrm{n}=53)\end{array}$ \\
\hline Direct sales & $\begin{array}{l}1.5 \% \\
(n=1)\end{array}$ & $\begin{array}{l}0.6 \% \\
(n=1)\end{array}$ & $\begin{array}{l}1.4 \% \\
(n=1)\end{array}$ & $\begin{array}{l}0.8 \% \\
(n=2)\end{array}$ & $\begin{array}{l}2.4 \% \\
(n=1)\end{array}$ & $\begin{array}{c}0.8 \% \\
(n=1)\end{array}$ & $\begin{array}{l}1.1 \% \\
(n=2)\end{array}$ \\
\hline $\begin{array}{c}\text { Another answer: online } \\
\text { pharmacy }\end{array}$ & $\begin{array}{l}1.5 \% \\
(n=1)\end{array}$ & $\begin{array}{c}0.0 \% \\
(\mathrm{n}=0)\end{array}$ & $\begin{array}{l}0.0 \% \\
(\mathrm{n}=0)\end{array}$ & $\begin{array}{l}0.4 \% \\
(\mathrm{n}=1)\end{array}$ & $\begin{array}{c}0.0 \% \\
(\mathrm{n}=0)\end{array}$ & $\begin{array}{c}0.0 \% \\
(\mathrm{n}=0)\end{array}$ & $\begin{array}{l}0.6 \% \\
(n=1)\end{array}$ \\
\hline Not applicable & $\begin{array}{c}9.1 \% \\
(n=6)\end{array}$ & $\begin{array}{c}5.6 \% \\
(n=9)\end{array}$ & $\begin{array}{l}2.7 \% \\
(n=2)\end{array}$ & $\begin{array}{c}4.3 \% \\
(n=11)\end{array}$ & $\begin{array}{l}14.3 \% \\
(n=6)\end{array}$ & $\begin{array}{l}5.0 \% \\
(n=6)\end{array}$ & $\begin{array}{c}6.1 \% \\
(n=11)\end{array}$ \\
\hline \multicolumn{8}{|c|}{ Question: What form of dietary supplements do you choose most often? } \\
\hline Tablets & $\begin{array}{l}75.8 \% \\
(n=50)\end{array}$ & $\begin{array}{c}77.5 \% \\
(n=124)\end{array}$ & $\begin{array}{c}81.1 \% \\
(n=60)\end{array}$ & $\begin{array}{c}81.4 \% \\
(n=210)\end{array}$ & $\begin{array}{l}57.1 \% \\
(n=24)\end{array}$ & $\begin{array}{c}80.2 \% \\
(n=97)\end{array}$ & $\begin{array}{c}76.5 \% \\
(n=137)\end{array}$ \\
\hline Capsules & $\begin{array}{l}54.5 \% \\
(n=36)\end{array}$ & $\begin{array}{l}60.0 \% \\
(n=96)\end{array}$ & $\begin{array}{l}56.8 \% \\
(n=42)\end{array}$ & $\begin{array}{c}58.1 \% \\
(n=150)\end{array}$ & $\begin{array}{c}57.1 \% \\
(n=24)\end{array}$ & $\begin{array}{c}57.0 \% \\
(n=69)\end{array}$ & $\begin{array}{c}58.7 \% \\
(\mathrm{n}=105)\end{array}$ \\
\hline Effervescent tablets & $\begin{array}{l}13.6 \% \\
(n=9)\end{array}$ & $\begin{array}{c}12.5 \% \\
(n=20)\end{array}$ & $\begin{array}{l}12.2 \% \\
(n=9)\end{array}$ & $\begin{array}{c}12.0 \% \\
(\mathrm{n}=31)\end{array}$ & $\begin{array}{l}16.7 \% \\
(n=7)\end{array}$ & $\begin{array}{c}17.4 \% \\
(n=21)\end{array}$ & $\begin{array}{c}9.5 \% \\
(n=17)\end{array}$ \\
\hline Dissolving sachets & $\begin{array}{c}7.6 \% \\
(n=5)\end{array}$ & $\begin{array}{c}6.3 \% \\
(n=10)\end{array}$ & $\begin{array}{c}14.9 \% \\
(n=11)\end{array}$ & $\begin{array}{c}7.8 \% \\
(n=20)\end{array}$ & $\begin{array}{l}14.3 \% \\
(n=6)\end{array}$ & $\begin{array}{c}8.3 \% \\
(n=10)\end{array}$ & $\begin{array}{c}8.9 \% \\
(n=16)\end{array}$ \\
\hline
\end{tabular}


Table 3. Dietary supplements in the opinion of students of three faculties of the Medical University in Białystok (cont.).

\begin{tabular}{|c|c|c|c|c|c|c|c|}
\hline Division & \multicolumn{3}{|c|}{ Faculty } & \multicolumn{2}{|c|}{ Gender } & \multicolumn{2}{|c|}{ Period of study } \\
\hline Parameters & $\begin{array}{c}\% \text { of FM } \\
\text { students } \\
(n=66)\end{array}$ & $\begin{array}{l}\% \text { of FP } \\
\text { students } \\
(\mathrm{n}=160)\end{array}$ & $\begin{array}{c}\% \text { of FHS } \\
\text { students } \\
(\mathrm{n}=74)\end{array}$ & $\begin{array}{c}\% \text { of } \\
\text { women } \\
(\mathrm{W}) \\
(\mathrm{n}=258)\end{array}$ & $\begin{array}{c}\% \text { of men } \\
(\mathrm{M}) \\
(\mathrm{n}=42)\end{array}$ & $\begin{array}{l}\quad \% \text { of } \\
\text { students on } \\
\text { I-III years } \\
\text { (I group) } \\
(\mathrm{n}=121)\end{array}$ & $\begin{array}{c}\% \text { of students } \\
\text { of subsequent } \\
\text { years of study } \\
\text { (II group) } \\
(\mathrm{n}=179)\end{array}$ \\
\hline Spray & $\begin{array}{c}0.0 \% \\
(\mathrm{n}=0)\end{array}$ & $\begin{array}{c}0.6 \% \\
(\mathrm{n}=1)\end{array}$ & $\begin{array}{c}0.0 \% \\
(\mathrm{n}=0)\end{array}$ & $\begin{array}{c}0.4 \% \\
(\mathrm{n}=1)\end{array}$ & $\begin{array}{c}0.0 \% \\
(\mathrm{n}=0)\end{array}$ & $\begin{array}{c}0.8 \% \\
(\mathrm{n}=1)\end{array}$ & $\begin{array}{c}0.0 \% \\
(\mathrm{n}=0)\end{array}$ \\
\hline Drops & $\begin{array}{c}1.5 \% \\
(\mathrm{n}=1)\end{array}$ & $\begin{array}{c}8.8 \% \\
(\mathrm{n}=14)\end{array}$ & $\begin{array}{c}2.7 \% \\
(\mathrm{n}=2)\end{array}$ & $\begin{array}{c}6.2 \% \\
(\mathrm{n}=16)\end{array}$ & $\begin{array}{c}2.4 \% \\
(\mathrm{n}=1)\end{array}$ & $\begin{array}{l}2.5 \% \\
(\mathrm{n}=3)\end{array}$ & $\begin{array}{c}7.8 \% \\
(n=14)\end{array}$ \\
\hline Lozenges & $\begin{array}{c}1.5 \% \\
(\mathrm{n}=1)\end{array}$ & $\begin{array}{c}3.1 \% \\
(\mathrm{n}=5)\end{array}$ & $\begin{array}{c}1.4 \% \\
(\mathrm{n}=1)\end{array}$ & $\begin{array}{c}2.3 \% \\
(\mathrm{n}=6)\end{array}$ & $\begin{array}{c}2.4 \% \\
(\mathrm{n}=1)\end{array}$ & $\begin{array}{c}5.0 \% \\
(\mathrm{n}=6)\end{array}$ & $\begin{array}{c}0.6 \% \\
(\mathrm{n}=1)\end{array}$ \\
\hline Syrups & $\begin{array}{c}1.5 \% \\
(\mathrm{n}=1)\end{array}$ & $\begin{array}{c}1.3 \% \\
(\mathrm{n}=2)\end{array}$ & $\begin{array}{c}0.0 \% \\
(\mathrm{n}=0)\end{array}$ & $\begin{array}{c}1.2 \% \\
(\mathrm{n}=3)\end{array}$ & $\begin{array}{c}0.0 \% \\
(\mathrm{n}=0)\end{array}$ & $\begin{array}{c}0.8 \% \\
(\mathrm{n}=1)\end{array}$ & $\begin{array}{c}1.1 \% \\
(\mathrm{n}=2)\end{array}$ \\
\hline Lollipops & $\begin{array}{l}0.0 \% \\
(\mathrm{n}=0)\end{array}$ & $\begin{array}{c}0.0 \% \\
(\mathrm{n}=0)\end{array}$ & $\begin{array}{c}1.4 \% \\
(\mathrm{n}=1)\end{array}$ & $\begin{array}{c}0.4 \% \\
(\mathrm{n}=1)\end{array}$ & $\begin{array}{c}0.0 \% \\
(\mathrm{n}=0)\end{array}$ & $\begin{array}{c}0.8 \% \\
(\mathrm{n}=1)\end{array}$ & $\begin{array}{c}0.0 \% \\
(\mathrm{n}=0)\end{array}$ \\
\hline Jelly beans & $\begin{array}{c}0.0 \% \\
(\mathrm{n}=0)\end{array}$ & $\begin{array}{c}1.3 \% \\
(\mathrm{n}=2)\end{array}$ & $\begin{array}{c}1.4 \% \\
(\mathrm{n}=1)\end{array}$ & $\begin{array}{c}1.2 \% \\
(\mathrm{n}=3)\end{array}$ & $\begin{array}{c}0.0 \% \\
(\mathrm{n}=0)\end{array}$ & $\begin{array}{c}2.5 \% \\
(\mathrm{n}=3)\end{array}$ & $\begin{array}{c}0.0 \% \\
(\mathrm{n}=0)\end{array}$ \\
\hline $\begin{array}{c}\text { Other answer - total: } \\
\text { - liquid, } \\
\text { - Whey protein concentrate }\end{array}$ & $\begin{array}{l}3.0 \% \\
(\mathrm{n}=2)\end{array}$ & $\begin{array}{c}0.0 \% \\
(\mathrm{n}=0)\end{array}$ & $\begin{array}{c}0.0 \% \\
(\mathrm{n}=0)\end{array}$ & $\begin{array}{c}0.4 \% \\
(\mathrm{n}=1)\end{array}$ & $\begin{array}{c}2.4 \% \\
(\mathrm{n}=1)\end{array}$ & $\begin{array}{c}0.0 \% \\
(\mathrm{n}=0)\end{array}$ & $\begin{array}{c}1.2 \% \\
(\mathrm{n}=2)\end{array}$ \\
\hline Not applicable & $\begin{array}{c}7.6 \% \\
(n=5)\end{array}$ & $\begin{array}{l}3.1 \% \\
(\mathrm{n}=5)\end{array}$ & $\begin{array}{l}2.7 \% \\
(\mathrm{n}=2)\end{array}$ & $\begin{array}{c}3.1 \% \\
(\mathrm{n}=8)\end{array}$ & $\begin{array}{l}9.5 \% \\
(\mathrm{n}=4)\end{array}$ & $\begin{array}{c}3.3 \% \\
(\mathrm{n}=4)\end{array}$ & $\begin{array}{c}4.7 \% \\
(\mathrm{n}=8)\end{array}$ \\
\hline \multicolumn{8}{|c|}{ Question: If the dietary supplement you use is not available, do you buy any substitute? } \\
\hline Yes & $\begin{array}{c}60.6 \% \\
(\mathrm{n}=40)^{* *}\end{array}$ & $\begin{array}{c}40.0 \% \\
(n=64)\end{array}$ & $\begin{array}{c}35.1 \% \\
(\mathrm{n}=26)\end{array}$ & $\begin{array}{c}44.6 \% \\
(\mathrm{n}=115)^{* *}\end{array}$ & $\begin{array}{c}35.7 \% \\
(\mathrm{n}=15)\end{array}$ & $\begin{array}{c}52.9 \% \\
(\mathrm{n}=64)^{*}\end{array}$ & $\begin{array}{c}36.9 \% \\
(\mathrm{n}=66)\end{array}$ \\
\hline No & $\begin{array}{c}24.2 \% \\
(\mathrm{n}=16)\end{array}$ & $\begin{array}{c}34.4 \% \\
(\mathrm{n}=55)\end{array}$ & $\begin{array}{c}47.3 \% \\
(n=35)\end{array}$ & $\begin{array}{c}37.2 \% \\
(\mathrm{n}=96)\end{array}$ & $\begin{array}{c}23.1 \% \\
(\mathrm{n}=10)\end{array}$ & $\begin{array}{c}31.4 \% \\
(\mathrm{n}=38)\end{array}$ & $\begin{array}{c}38.0 \% \\
(\mathrm{n}=68)\end{array}$ \\
\hline Not applicable & $\begin{array}{c}15.2 \% \\
(\mathrm{n}=10)\end{array}$ & $\begin{array}{c}25.6 \% \\
(\mathrm{n}=41)\end{array}$ & $\begin{array}{c}17.6 \% \\
(n=13)\end{array}$ & $\begin{array}{c}18.2 \% \\
(\mathrm{n}=47)\end{array}$ & $\begin{array}{c}41.2 \% \\
(n=17)\end{array}$ & $\begin{array}{c}15.7 \% \\
(\mathrm{n}=19)\end{array}$ & $\begin{array}{c}25.1 \% \\
(\mathrm{n}=45)\end{array}$ \\
\hline \multicolumn{8}{|c|}{ Question: In your opinion, are dietary supplements tested before being placed on the market? } \\
\hline Yes & $\begin{array}{c}40.9 \% \\
(\mathrm{n}=27)\end{array}$ & $\begin{array}{c}38.8 \% \\
(n=62)\end{array}$ & $\begin{array}{c}54.0 \% \\
(\mathrm{n}=40)\end{array}$ & $\begin{array}{c}42.6 \% \\
(\mathrm{n}=110)\end{array}$ & $\begin{array}{c}45.2 \% \\
(n=19)\end{array}$ & $\begin{array}{c}62.0 \% \\
(\mathrm{n}=75) \\
\end{array}$ & $\begin{array}{c}30.2 \% \\
(\mathrm{n}=54) \\
\end{array}$ \\
\hline No & $\begin{array}{c}59.1 \% \\
(\mathrm{n}=39)\end{array}$ & $\begin{array}{c}61.2 \% \\
(\mathrm{n}=98)\end{array}$ & $\begin{array}{c}46.0 \% \\
(n=34)\end{array}$ & $\begin{array}{c}57.4 \% \\
(\mathrm{n}=148)\end{array}$ & $\begin{array}{c}54.8 \% \\
(\mathrm{n}=23)\end{array}$ & $\begin{array}{c}38.0 \% \\
(n=46)\end{array}$ & $\begin{array}{c}69.8 \% \\
(\mathrm{n}=125)^{* * *}\end{array}$ \\
\hline
\end{tabular}

FHS - Faculty of Health Sciences, FM - Faculty of Medicine with the Division of Dentistry and Division of Medical Education in English, FP - Faculty of Pharmacy with the Division of Laboratory Medicine, ${ }^{*} \mathrm{p}<0.05,{ }^{* *} \mathrm{p}<0.01,{ }^{* * *} \mathrm{p}<0.001$

The factors that guide the respondents when choosing a dietary supplement were analyzed. One of the most important criteria is composition $(77.3 \%, 90.0 \%$, and $71.6 \%$ of students respectively). The second important factor is the price $(40.9 \%, 37.5 \%$, and $43.2 \%)$. The recommendations of pharmacists $(39.4 \%, 43.8 \%$, and $24.3 \%$ ) and doctors $(39.4 \%, 20.6 \%$, and $31.1 \%$ ) are also important for students. Interestingly, advertising influences the purchasing decisions of a very small group of students $(0.0 \%, 2.5 \%$, and $6.8 \%)$. Moreover, significant differences were demonstrated in the criteria that men and women follow when making decisions about the purchase of dietary supplements. Representatives of both sexes agreed on such criteria as price $(39.9 \%$ vs. $38.1 \%)$ and composition
(77.5\% vs. $76.2 \%$ ), but women listened to pharmacists' recommendations significantly more often (40.3\% vs. $23.9 \%)$.

Significant differences were also shown in the case of the source of knowledge about dietary supplements. The most important source is the Internet (indicated by $66.7 \%, 65.6 \%$, and $73.0 \%$ of students). In the case of students of the MUB, studies are the second most important source of knowledge (FM: $57.6 \%$ of the respondents, FP: $80.6 \%$, FHS: $43.2 \%$ ). Pharmacists and doctors are also an important source of knowledge (FM: $37.9 \%$, and $42.4 \%$, FP: $41.3 \%$, and $19.4 \%$, PHS: $20.3 \%$, and $18.9 \%$ ). Men more often indicated the Internet as the main source of knowledge about dietary supplements (81.0\% vs. $65.5 \%)$, however, the percentage of people indicating that 
studying was one of the main sources of knowledge was similar for both gender (W: $66.7 \%$ vs. M: $64.3 \%$ ). Students with more experience ( $4^{\text {th }}$ year and higher) significantly more often indicated classes during their studies as the greatest source of knowledge about dietary supplements $(76.5 \%$ vs. $51.2 \%)$.

A beneficial effect resulting from the use of dietary supplements was noted by slightly more than half of the respondents (FM: $56.1 \%$, FP: $54.4 \%$, FHS: $55.4 \%$ ). Side effects resulting from the use of dietary supplements were reported only by a small group of respondents (FM: 4.5\%, FP: 3.7\%, FHS: $2.7 \%$ ), while the regular use of dietary supplements was declared by approximately half of the respondents (FM: $56.1 \%$, FP: 39.4\%, FHS: 51.4\%). Women more often reported beneficial effects resulting from the use of dietary supplements (W: $56.9 \%$ vs. M: $45.2 \%$ ), but it was men who reported side effects more often (M: $4.8 \%$ vs. $3.5 \%$ ). Almost half of the women declared regular use of dietary supplements (W: $47.3 \%$ vs. M: $38.1 \%)$.

Among students with FM, students spend an average of PLN 10 to 19 (30.3\%) or PLN 20 to 40 (27.3\%) per month. The highest percentage of FP students spends between PLN 20 and PLN 40 (32.5\%), similar to FHS students (35.1\%). It has been shown that pharmacies are the main place to buy dietary supplements (FM: $80.3 \%$, FP: $86.9 \%$, FHS: $87.8 \%$ ). The second and most frequently indicated place of purchase is the Internet (FM: $19.7 \%$, FP: $25.6 \%$, FHS: $31.1 \%$ ), therefore dietary supplements in the non-pharmacy trade should also be of good quality.
Students most often buy dietary supplements in the form of tablets and capsules (respectively: $75.8 \%$ and $54.5 \%$ of students with FM, $77.5 \%$ and $60.0 \%$ with FP, $81.1 \%$, and $56.8 \%$ with FHS).

Significant differences were indicated by analyzing the answers to the question regarding the purchase of a substitute if the preparation the student wants to buy is not available for sale. Most students with FM choose a substitute $(60.6 \%)$, in the case of students with FP and FHS it is a smaller percentage $(40.0 \%$ and $35.1 \%)$, which indicates attachment to the dietary supplements used. In the absence of a specific dietary supplement on sale, preparations of the same composition are used significantly more often by women than men $(44.6 \%)$ and significantly more often by students of the initial period of study (52.9\%).

The attachment to the use of specific dietary supplements may result from the belief that some of them are tested before being marketed. It has been shown that the highest percentage of FHS students say that dietary supplements are tested (54.0\%). Only $40.9 \%$ of FM students and $38.8 \%$ of FP students were of a similar opinion. Moreover, it was shown that significantly more students of older years of studies (IV year and above, 69.8\%) assess that dietary supplements are not tested before they are put on sale.

Table 4 presents data on students' opinions on marketing activities. Students named TV commercials as having the greatest chance of reaching their audience (FM: 72.7\%, FP: 78.8\%, and FHS: 85.1\%). Online advertising came second (FM: $51.5 \%$, FP: $57.5 \%$, FHS: $60.8 \%$ ). Most of the students judged

Table 4. Students' opinions on the marketing of dietary supplements.

\begin{tabular}{|c|c|c|c|c|c|c|c|}
\hline Division & \multicolumn{3}{|c|}{ Faculty } & \multicolumn{2}{|c|}{ Gender } & \multicolumn{2}{|c|}{ Period of study } \\
\hline Parameters & $\begin{array}{c}\% \text { of FM } \\
\text { students } \\
(n=66)\end{array}$ & $\begin{array}{l}\% \text { of FP } \\
\text { students } \\
(n=160)\end{array}$ & $\begin{array}{c}\% \text { of FHS } \\
\text { students } \\
(\mathrm{n}=74)\end{array}$ & $\begin{array}{c}\% \text { of } \\
\text { women } \\
(\mathrm{W}) \\
(\mathrm{n}=258)\end{array}$ & $\begin{array}{c}\% \text { of men } \\
(\mathrm{M}) \\
(\mathrm{n}=42)\end{array}$ & $\begin{array}{l}\% \text { of students } \\
\text { on I-III years } \\
\text { (I group) } \\
(\mathrm{n}=121)\end{array}$ & $\begin{array}{l}\% \text { of students of } \\
\text { subsequent years } \\
\text { of study } \\
\text { (II group) } \\
(\mathrm{n}=179)\end{array}$ \\
\hline \multicolumn{8}{|c|}{ Question: Which ads do you think have the best chance of reaching audience? } \\
\hline On the Internet & $\begin{array}{l}51.5 \% \\
(\mathrm{n}=34)\end{array}$ & $\begin{array}{l}57.5 \% \\
(n=92)\end{array}$ & $\begin{array}{l}60.8 \% \\
(n=45)\end{array}$ & $\begin{array}{c}57.0 \% \\
(n=147)\end{array}$ & $\begin{array}{c}57.1 \% \\
(\mathrm{n}=24)\end{array}$ & $\begin{array}{l}59.5 \% \\
(n=72)\end{array}$ & $\begin{array}{l}55.3 \% \\
(n=99)\end{array}$ \\
\hline In the press & $\begin{array}{c}1.5 \% \\
(\mathrm{n}=1)\end{array}$ & $\begin{array}{l}1.3 \% \\
(n=2)\end{array}$ & $\begin{array}{l}1.4 \% \\
(\mathrm{n}=1)\end{array}$ & $\begin{array}{c}1.6 \% \\
(n=4)\end{array}$ & $\begin{array}{c}0.0 \% \\
(\mathrm{n}=0)\end{array}$ & $\begin{array}{c}0.8 \% \\
(\mathrm{n}=1)\end{array}$ & $\begin{array}{c}1.7 \% \\
(n=3)\end{array}$ \\
\hline In TV & $\begin{array}{l}72.7 \% \\
(n=48)\end{array}$ & $\begin{array}{c}78.8 \% \\
(n=126)\end{array}$ & $\begin{array}{l}85.1 \% \\
(n=63)\end{array}$ & $\begin{array}{c}79.8 \% \\
(n=206)\end{array}$ & $\begin{array}{l}73.8 \% \\
(n=31)\end{array}$ & $\begin{array}{c}82.6 \% \\
(n=100)\end{array}$ & $\begin{array}{c}76.5 \% \\
(n=137)\end{array}$ \\
\hline \multicolumn{8}{|c|}{ Question: In your opinion, is the number of advertisements for dietary supplements adequate? } \\
\hline $\begin{array}{l}\text { Yes, I like watching } \\
\text { supplement ads and } \\
\text { hearing about new } \\
\text { preparations }\end{array}$ & $\begin{array}{l}3.0 \% \\
(\mathrm{n}=2)\end{array}$ & $\begin{array}{c}6.9 \% \\
(\mathrm{n}=11)\end{array}$ & $\begin{array}{l}12.2 \% \\
(\mathrm{n}=9)\end{array}$ & $\begin{array}{c}7.0 \% \\
(n=18)\end{array}$ & $\begin{array}{c}9.5 \% \\
(n=4)\end{array}$ & $\begin{array}{c}7.4 \% \\
(n=9)\end{array}$ & $\begin{array}{c}7.3 \% \\
(n=13)\end{array}$ \\
\hline $\begin{array}{l}\text { No, there are far too } \\
\text { many of them }\end{array}$ & $\begin{array}{c}97.0 \% \\
(n=64)\end{array}$ & $\begin{array}{c}93.1 \% \\
(\mathrm{n}=149)\end{array}$ & $\begin{array}{l}87.8 \% \\
(n=65)\end{array}$ & $\begin{array}{c}93.0 \% \\
(n=240)\end{array}$ & $\begin{array}{c}90.5 \% \\
(n=38)\end{array}$ & $\begin{array}{c}92.6 \% \\
(n=112)\end{array}$ & $\begin{array}{c}92.7 \% \\
(\mathrm{n}=166)\end{array}$ \\
\hline
\end{tabular}


Table 4. Students' opinions on the marketing of dietary supplements (cont.).

\begin{tabular}{|c|c|c|c|c|c|c|c|}
\hline Division & \multicolumn{3}{|c|}{ Faculty } & \multicolumn{2}{|c|}{ Gender } & \multicolumn{2}{|c|}{ Period of study } \\
\hline Parameters & $\begin{array}{l}\% \text { of FM } \\
\text { students } \\
(n=66)\end{array}$ & $\begin{array}{l}\% \text { of FP } \\
\text { students } \\
(n=160)\end{array}$ & $\begin{array}{c}\% \text { of FHS } \\
\text { students } \\
(n=74)\end{array}$ & $\begin{array}{c}\% \text { of } \\
\text { women } \\
(\mathrm{W}) \\
(\mathrm{n}=258)\end{array}$ & $\begin{array}{c}\% \text { of men } \\
(\mathrm{M}) \\
(\mathrm{n}=42)\end{array}$ & $\begin{array}{c}\% \text { of students } \\
\text { on I-III years } \\
(\text { I group) } \\
(\mathrm{n}=121)\end{array}$ & $\begin{array}{c}\% \text { of students of } \\
\text { subsequent years } \\
\text { of study } \\
\text { (II group) } \\
\text { (n = 179) }\end{array}$ \\
\hline \multicolumn{8}{|c|}{ Question: Do you use dietary supplements from own pharmacy brand? } \\
\hline Yes & $\begin{array}{l}25.8 \% \\
(n=17)\end{array}$ & $\begin{array}{l}16.3 \% \\
(n=26)\end{array}$ & $\begin{array}{l}10.8 \% \\
(n=8)\end{array}$ & $\begin{array}{c}17.4 \% \\
(n=45)\end{array}$ & $\begin{array}{l}14.3 \% \\
(n=6)\end{array}$ & $\begin{array}{l}20.6 \% \\
(n=25)\end{array}$ & $\begin{array}{l}14.5 \% \\
(n=26)\end{array}$ \\
\hline No & $\begin{array}{l}74.2 \% \\
(n=49)\end{array}$ & $\begin{array}{c}83.7 \% \\
(n=134)\end{array}$ & $\begin{array}{c}89.2 \% \\
(\mathrm{n}=66)^{*}\end{array}$ & $\begin{array}{c}82.6 \% \\
(n=213)\end{array}$ & $\begin{array}{l}85.7 \% \\
(n=36)\end{array}$ & $\begin{array}{c}79.4 \% \\
(n=96)\end{array}$ & $\begin{array}{c}85.5 \% \\
(n=153)\end{array}$ \\
\hline \multicolumn{8}{|c|}{ Question: Do you buy dietary supplements offered under direct marketing? } \\
\hline Yes & $\begin{array}{l}3.0 \% \\
(\mathrm{n}=2)\end{array}$ & $\begin{array}{c}6.3 \% \\
(n=10)\end{array}$ & $\begin{array}{c}4.1 \% \\
(n=3)\end{array}$ & $\begin{array}{c}5.4 \% \\
(n=14)\end{array}$ & $\begin{array}{l}2.4 \% \\
(\mathrm{n}=1)\end{array}$ & $\begin{array}{l}5.8 \% \\
(n=7)\end{array}$ & $\begin{array}{l}4.5 \% \\
(n=8)\end{array}$ \\
\hline No & $\begin{array}{c}97.0 \% \\
(n=64)\end{array}$ & $\begin{array}{c}91.8 \% \\
(\mathrm{n}=147)\end{array}$ & $\begin{array}{l}95.9 \% \\
(n=71)\end{array}$ & $\begin{array}{c}93.4 \% \\
(\mathrm{n}=241)\end{array}$ & $\begin{array}{l}97.6 \% \\
(n=41)\end{array}$ & $\begin{array}{c}93.4 \% \\
(n=113)\end{array}$ & $\begin{array}{c}94.4 \% \\
(n=169)\end{array}$ \\
\hline $\begin{array}{l}\text { I sell this type of } \\
\text { preparations myself }\end{array}$ & $\begin{array}{c}0.0 \% \\
(\mathrm{n}=0)\end{array}$ & $\begin{array}{l}1.9 \% \\
(n=3)\end{array}$ & $\begin{array}{l}0.0 \% \\
(\mathrm{n}=0)\end{array}$ & $\begin{array}{l}1.2 \% \\
(\mathrm{n}=3)\end{array}$ & $\begin{array}{c}0.0 \% \\
(\mathrm{n}=0)\end{array}$ & $\begin{array}{l}0.8 \% \\
(\mathrm{n}=1)\end{array}$ & $\begin{array}{l}1.1 \% \\
(n=2)\end{array}$ \\
\hline \multicolumn{8}{|c|}{ Question: Which preparation will you choose (taking into account only the price and advertising criteria)? } \\
\hline Cheaper but unknown & $\begin{array}{l}66.7 \% \\
(n=44)\end{array}$ & $\begin{array}{c}61.3 \% \\
(n=98)\end{array}$ & $\begin{array}{l}73.0 \% \\
(n=54)\end{array}$ & $\begin{array}{c}67.1 \% \\
(n=173)\end{array}$ & $\begin{array}{l}54.8 \% \\
(n=23)\end{array}$ & $\begin{array}{l}62.8 \% \\
(n=76)\end{array}$ & $\begin{array}{c}67.0 \% \\
(n=120)\end{array}$ \\
\hline $\begin{array}{l}\text { More expensive but } \\
\text { advertised }\end{array}$ & $\begin{array}{l}33.3 \% \\
(n=22)\end{array}$ & $\begin{array}{l}38.7 \% \\
(n=62)\end{array}$ & $\begin{array}{l}27.0 \% \\
(n=20)\end{array}$ & $\begin{array}{l}32.9 \% \\
(n=85)\end{array}$ & $\begin{array}{c}45.2 \% \\
(n=19)\end{array}$ & $\begin{array}{l}37.2 \% \\
(n=45)\end{array}$ & $\begin{array}{l}33.0 \% \\
(n=59)\end{array}$ \\
\hline \multicolumn{8}{|c|}{ Question: What is, in your opinion, the most effective promotional activity? } \\
\hline $\begin{array}{l}\text { Word of mouth } \\
\text { marketing }\end{array}$ & $\begin{array}{c}45.5 \% \\
(n=30) \\
\end{array}$ & $\begin{array}{l}51.3 \% \\
(\mathrm{n}=82)\end{array}$ & $\begin{array}{l}43.2 \% \\
(n=32)\end{array}$ & $\begin{array}{c}48.1 \% \\
(n=124)\end{array}$ & $\begin{array}{l}47.6 \% \\
(n=20)\end{array}$ & $\begin{array}{c}46.3 \% \\
(n=56)\end{array}$ & $\begin{array}{c}49.2 \% \\
(n=88)\end{array}$ \\
\hline $\begin{array}{l}\text { Advertising on } \\
\text { television }\end{array}$ & $\begin{array}{l}57.6 \% \\
(n=38)\end{array}$ & $\begin{array}{c}63.8 \% \\
(n=102)\end{array}$ & $\begin{array}{l}58.1 \% \\
(n=43)\end{array}$ & $\begin{array}{c}62.4 \% \\
(\mathrm{n}=161)\end{array}$ & $\begin{array}{l}52.4 \% \\
(n=22)\end{array}$ & $\begin{array}{l}62.8 \% \\
(n=76)\end{array}$ & $\begin{array}{c}59.8 \% \\
(n=107)\end{array}$ \\
\hline Internet advertising & $\begin{array}{l}37.9 \% \\
(\mathrm{n}=25) \\
\end{array}$ & $\begin{array}{l}56.9 \% \\
(\mathrm{n}=91)\end{array}$ & $\begin{array}{l}48.6 \% \\
(n=36)\end{array}$ & $\begin{array}{c}49.6 \% \\
(n=128)\end{array}$ & $\begin{array}{c}57.1 \% \\
(\mathrm{n}=24) \\
\end{array}$ & $\begin{array}{c}52.0 \% \\
(\mathrm{n}=63) \\
\end{array}$ & $\begin{array}{c}49.7 \% \\
(n=89) \\
\end{array}$ \\
\hline Advertising in the press & $\begin{array}{l}1.5 \% \\
(n=1)\end{array}$ & $\begin{array}{l}4.4 \% \\
(n=7)\end{array}$ & $\begin{array}{l}2.7 \% \\
(n=2)\end{array}$ & $\begin{array}{l}3.5 \% \\
(n=9)\end{array}$ & $\begin{array}{l}2.4 \% \\
(n=1)\end{array}$ & $\begin{array}{l}0.0 \% \\
(n=0)\end{array}$ & $\begin{array}{c}5.6 \% \\
(n=10)\end{array}$ \\
\hline $\begin{array}{l}\text { Other answer - total: } \\
\text { - radio advertising, } \\
\text { - doctor, } \\
\text { - good product quality } \\
\text { and competitive price, } \\
\text { - advertising in } \\
\text { social media and by } \\
\text { influencers, } \\
\text { - free samples, } \\
\text { - I have no opinion. }\end{array}$ & $\begin{array}{l}10.6 \% \\
(n=7)\end{array}$ & $\begin{array}{c}3.7 \% \\
(n=5)\end{array}$ & $\begin{array}{c}1.4 \% \\
(n=1)\end{array}$ & $\begin{array}{c}5.1 \% \\
(n=13)\end{array}$ & $\begin{array}{c}0.0 \% \\
(\mathrm{n}=0)\end{array}$ & $\begin{array}{c}2.5 \% \\
(n=3)\end{array}$ & $\begin{array}{c}5.6 \% \\
(n=10)\end{array}$ \\
\hline
\end{tabular}

FHS - Faculty of Health Sciences, FM - Faculty of Medicine with the Division of Dentistry and Division of Medical Education in English, FP - Faculty of Pharmacy with the Division of Laboratory Medicine, * p $<0.05$

the number of ads too high (FM: $97.0 \%$, FP: $93.1 \%$, FHS: $87.8 \%$ ). Moreover, it was shown that a significantly higher percentage of FHS students do not use their own pharmacy brand dietary supplements (89.2\%) compared to $83.7 \%$ of FP students and $74.2 \%$ of FM students.

Despite the fact that most students previously indicated that the composition of supplements is the most important, about two-thirds of respondents indicated that they would choose a cheaper but unknown product (FM: 66.7\%, FP: 61.3\%, FHS: $73.0 \%$ ). The students indicated that the most effective marketing activities are: TV advertising (FM: 57.6\%, FP: $63.8 \%$, FHS: 58.1\%), Internet advertising (FM: $37.9 \%$, FP: $56.9 \%$, FHS: $48.6 \%$ ) and word of mouth marketing (FM: 45.5\%, FP: 51.3\%, FHS: $43.2 \%$ ). The lack of belief in the effectiveness of supplements of own brand of pharmacies 
is indicated by a similar percentage of representatives of both sexes: over $80 \%$ of respondents (W: $82.6 \%$ vs. M: $85.7 \%$ ), while over $90 \%$ do not buy dietary supplements offered under direct marketing (W: 93.4\% vs. M: 97.6\%). Women more often indicate that they would choose a cheaper but unknown supplement (67.1\% vs. 54.8\%). Women most often indicated TV advertisements as the most effective marketing activity (62.4\%), while men: Internet advertising (57.1\%).

Early years students more often indicated that television and Internet advertisements had a greater chance of reaching the audience (I: $82.6 \%$ and $59.5 \%$ vs. II: $76.5 \%$ and $55.3 \%$ ). Both groups of students agreed that in their opinion there were too many advertisements for dietary supplements (I: $92.6 \%$ vs. II: $92.7 \%$ ). Older students are characterized by a greater lack of trust in the dietary supplements of their own brands of pharmacies (II: $85.5 \%$ vs. I: $79.4 \%$ ). Over time, the percentage of people who use dietary supplements sold under direct marketing also decreases
(I: 5.8\% vs. II: 4.5\%). However, older students slightly more often indicate that they would buy a dietary supplement that is cheaper, but unknown (II: $67.0 \%$ vs. $62.8 \%$ ).

Moreover, it was shown that MUB students most often used dietary supplements containing vitamin D and / or vitamin K (as much as $35.33 \%$ ). The second most frequently chosen type of dietary supplements were preparations containing omega 3 and / or omega 6 and / or omega 9. In addition, $4 \%$ of students used supplements with zinc and $3.67 \%$ dietary supplements affecting the immunity (e.g. containing rutin) (Figure 1).

\section{DISCUSSION AND CONCLUSION}

The opinion of medical university students may result in the recommendation of specific dietary supplements for patients in future work or the lack of it, therefore the purpose of the analyzes was to evaluate

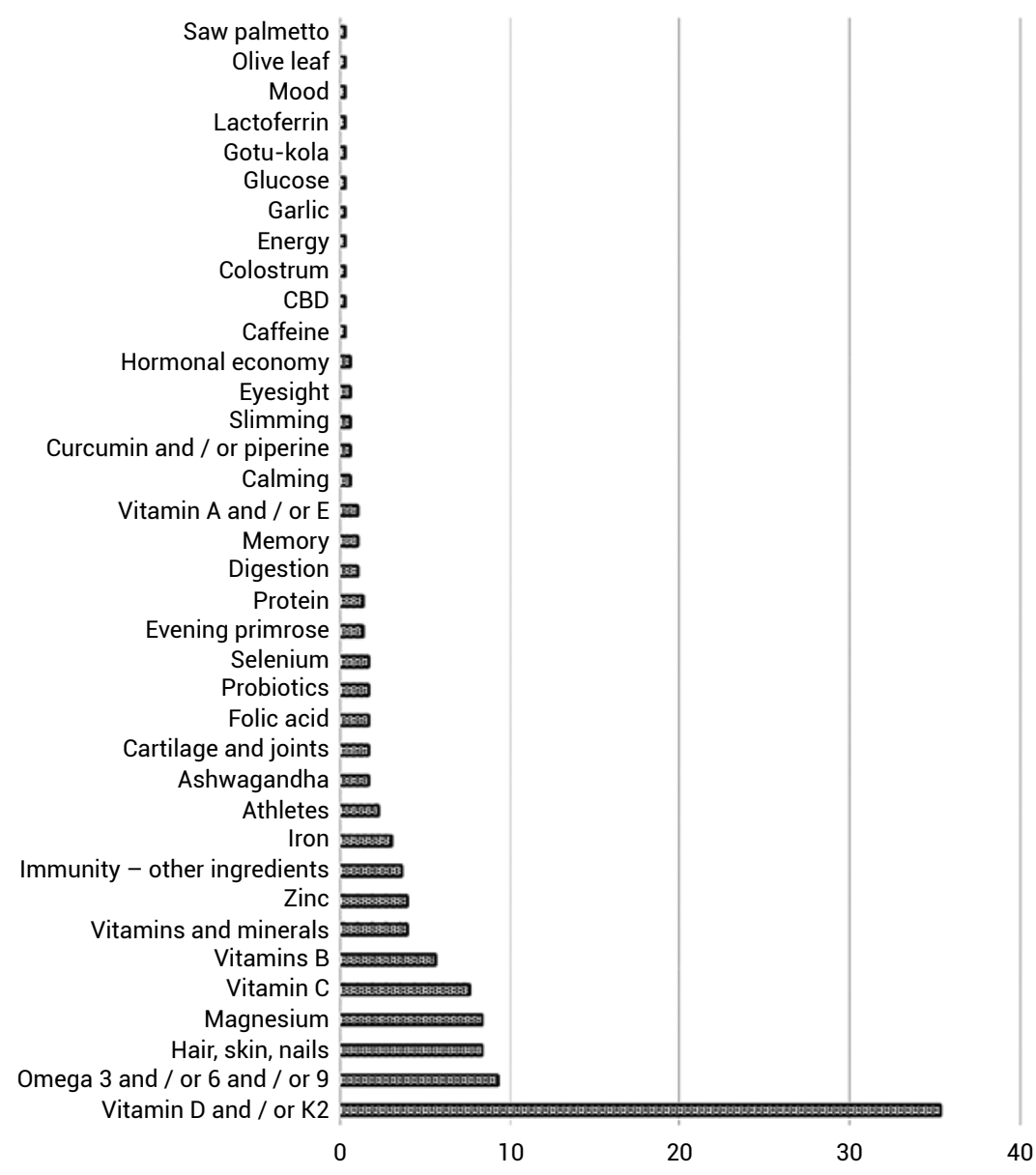

Figure 1. Ingredients or categories of dietary supplements used by students during this study (XI-XII 2020). 
these opinions on dietary supplements and marketing activities in this area.

This study showed that $56.1 \%$ of FM students, $39.4 \%$ of FP students, and $51.4 \%$ of FHS students declare regular use of dietary supplements. An analysis broken down by gender allowed to state that $47.3 \%$ of women and $38.1 \%$ of men declare regular use of dietary supplements. The results obtained by us are higher than those described by Sigłowa et al. (2009), who reported the consumption of dietary supplements by $38.3 \%$ of female students and $37.7 \%$ of male students (11).

Our earlier study, which aimed, inter alia, at the evaluation of the consumption of minerals along with the diet showed that $66 \%$ of 79 students of three fields of study (medical analytics, dietetics, and cosmetology) declared the use of dietary supplements during the last year. The most frequently used preparations were dietary supplements from the vitamin and mineral category (12).

Wilczewska et al. (2015), who assessed the knowledge of dietary supplements among selected students of the Medical University in Białystok and among students of post-secondary school, showed that there is a need for increased education in the field of dietary supplements. The authors showed that $21.9 \%$ of respondents used dietary supplements during the survey, and $53.7 \%$ used them earlier. The most frequently used group of supplements were vitamins and minerals (28.7\%). Surprisingly, as many as $39.4 \%$ of respondents, when asked about understanding the meaning of the term "dietary supplement", answered that it is difficult to say (13). In our study, when asked what a dietary supplement is, the correct answer was given by $77.3 \%$ of FM students, $77.5 \%$ of FP students, and $70.3 \%$ of FHS students.

Interesting research results, regarding the opinions of 153 pharmaceutical technicians, pharmacists, and doctors of pharmaceutical sciences, were published by Ratajczak et al. (2019). The opinions of this group of specialists were assessed on dietary supplements that improve mood and the factors that determine the purchase. It was shown that, in the opinion of these specialists, the most important factor determining the choice of a dietary supplement by patients is advertising (91\%), specialist advice ( $80 \%)$, and the opinion of friends and family (64\%). The information on internet forums $(20 \%)$ influences the decision to a much lesser extent, and what is worrying, only $12 \%$ of the composition and properties. The respondents also assessed that, according to patients, the most important features determining the purchase of a dietary supplement are the price $(93 \%)$ and the appearance of the packaging $(80 \%)$. These data indicate that the opinions of working people are more skeptical than of students (14).

Morris and Avorn (2003) evaluated internet marketing efforts for 8 herbal supplements in 5 popular web browsers. It was estimated that as many as $55 \%$ of websites contained information suggesting that supplements cure or help diagnose diseases (15). The information on internet portals must be true, as it is one of the most important media sources for students and patients.

Krejpcio et al. (2011) studied the use of dietary supplements by students of dietetics and psychology. They showed that $61 \%$ of dietetics students and $47 \%$ of psychology students declare taking dietary supplements, of which $15 \%$ of dietetics students and $15 \%$ of psychology students taking them every day. In our study, regular use of supplements was declared by $56.1 \%$ of FM students, $39.4 \%$ of FP students, and $51.4 \%$ of FHS students. Interestingly, only $12 \%$ of dietetics students and $15 \%$ of psychology students consulted a doctor about the consumption of dietary supplements (16).

Among the socio-demographic factors that affect the choice of a vitamin and mineral dietary supplement by adolescents up to 20 years of age, it is indicated, inter alia, higher level of mother's education and modification of the diet to make better nutritional decisions (17).

Our research showed that students of all three faculties buy dietary supplements primarily from a pharmacy: $80.3 \%$ of FM students, $86.9 \%$ of FP students, and $87.8 \%$ of FHS students. Only $3.0 \%$ of FM students, $7.5 \%$ of FP students, and $5.4 \%$ of FHS students buy dietary supplements in herbal and medical stores. The place of purchase is very important as it has been shown that in herbal and medical stores it is possible to obtain insufficient information about the products purchased $(18,19)$. It was estimated that in health food stores unscientific advice was received in $88 \%$ of cases, while in pharmacies - only in $27 \%$ of cases (19). Research published 5 years ago by Kowalik et al. (2016) showed a similar tendency among students to places where they buy dietary supplements: $85 \%$ of students indicated a pharmacy. Our research showed a different trend in purchasing dietary supplements: over the Internet (it was respectively: $19.7 \%$ of FM students, $25.6 \%$ of FP students, and $31.1 \%$ of FHS students, while the Kowalik study showed 10 times fewer students who use this form (20).

In order to increase profits from the sale of dietary supplements, pharmacies, herbal and medical stores, and among others, manufacturers implement various marketing activities. One of the main 
types of marketing is internet marketing. Reports are showing that websites do not contain all the necessary information about dietary supplements. For example, Bjelica et al. (2020) analyzed marketing activities regarding dietary supplements that protect the heart. The first 50 pages found by the search engines Google, Bing, and Yahoo were analyzed. It has been shown that the most frequently registered cardioprotective dietary supplements on the Internet were dietary supplements containing omega-3 acids (present on $64.05 \%$ of websites) (21). Importantly, information on side effects that may occur was indicated only on 1 website, which constituted $1.75 \%$ of the pages finally analyzed (in total: 89 ). Among the surveyed students whose responses were analyzed in our study, it was shown that side effects after dietary supplements occurred in $4.5 \%$ of students of FM, 3.7\% of students of FP, and $2.7 \%$ of students of FHS (Table 3). Information on potential side effects should be available on websites, in particular on the websites of pharmacies or herbal and medical stores. It is also important from the perspective of patients who use polypharmacy and drugs may interact with dietary supplements.

Research conducted by Ciszek and Duma shows that as many as $71.5 \%$ of students declare the use of dietary supplements. About half of this group (36.0\%) know the potential dangers of using them (22). Our studies showed that $3.5 \%$ of women and $4.8 \%$ of men reported side effects resulting from the use of dietary supplements, while the beneficial effects were noticed by $56.9 \%$ of women and $45.2 \%$ of the surveyed men.

Research conducted by Krejpcio et al. (2011) in a group of 50 physically active people aged 21 to 31 showed that only $40 \%$ of respondents knew the definition of a dietary supplement. Surprisingly, the occurrence of various ailments related to the consumption of supplements was declared by as many as $64 \%$ of the respondents, and despite this, as many as $78 \%$ of the respondents declared exceeding the recommended daily dose of supplements (23). Our research shows a greater awareness of the dosage and use of dietary supplements by students of medical universities. This is evidenced by the fact that only $3.5 \%$ of women and $4.8 \%$ of men reported side effects after using dietary supplements. The knowledge is also evidenced by the fact that $75.2 \%$ of students in the first three years and $76.0 \%$ of students in further years correctly indicated the definition of a dietary supplement.

As part of this work, we have shown that only $1.5 \%$ of FM students, $1.3 \%$ of FP, and $2.7 \%$ FHS students pay attention to the packaging. The packaging has not only a marketing function but also an informational, protective, logistic, ecological, and economic function (24). Research conducted in the United States carefully evaluates the packaging of vitamin preparations for children in terms of marketing, which may indicate that this population attaches more importance to this parameter. The authors analyzed 52 packages of supplements. Among the marketing activities aimed at children, the most frequently indicated were: the use of words related to taste and shape $(88.5 \%$ of packaging), while among the activities addressed to parents: description of body functions or structures $(82.7 \%)$ and expressions praising the lack of artificial flavors, colors, preservatives and sweeteners (also 82.7\%) (25).

It was shown that $1.9 \%$ of FP students sell dietary supplements under MLM (Multi-level Marketing). Opinions about this method of selling differ. For example, Cardenas and Fuchs-Tarlovsky assessed that this method of sale by doctors and nutritionists may be subject to a potential conflict of interest (26).

A study by Spiołek et al. (2011) showed that students most often use dietary supplements to improve the condition of hair, nails, and skin (as many as $75 \%$ of respondents), the second reason is convalescence after illness and preventive use of dietary supplements $(44.5 \%)$. In the above study, students of 4 fields of study (biotechnology, pharmacy, medical analytics, and cosmetology) also indicated what factors they take into account when choosing a vitamin and mineral preparation. Students primarily pay attention to the composition $(89 \%, 96 \%, 84 \%$ and $90 \%$ of students of the above-mentioned fields, respectively), then to the price $(51 \%, 31 \%, 37 \%$ and $46 \%)$ and the doctor's recommendation (19\%, 29\%, $24 \%$ and $14 \%$ ). Marketing considerations are taken into account by a small percentage of respondents: more students pay attention to advertising $(6 \%, 2 \%$, $10 \%$ and $28 \%)$ than to packaging $(2 \%, 2 \%, 0 \%$ and $0 \%$ ) (27). The obtained results, presenting the main factors, are in line with the results obtained in our study, according to which advertising is important only for $2.5 \%$ of FP students and $6.8 \%$ of FHS students, while $0.0 \%$ of FM students.

A favorable tendency, which we have shown in our research, is the fact that students of medical university obtain knowledge about dietary supplements mainly from the Internet and studies - in the case of FP students, studies were indicated by $80.6 \%$ of the respondents, and the Internet by $65.6 \%$. The Internet was also indicated by students in the study by Spiołek et al. (2011) as the main source of knowledge: it was 
$53 \%, 50 \%, 51 \%$, and $66 \%$ of students of the abovementioned fields, respectively (27).

Advertising is defined as a form of presentation and promotion of ideas, services, or goods, using e.g. press, transmission media, networks, media, and visual media, including through billboards and posters (7). Students are overwhelmed, for example, with the number of advertisements in advertising blocks, repeated advertisements for the same products, or being misled.

Lobb (2012) published a scientific fact-check that dealt with marketing claims made by mangosteen juice dietary supplements. He showed that many terms are imprecise and that the conclusions are too hasty. For example, marketing terms included the statement that the use of juice for 8 weeks lowers C-reactive protein levels. However, this study was conducted only on 40 participants, so it was not possible to detect side effects, e.g. with a frequency of $1 \%(28)$.

We showed that both among students in their first years and subsequent years, the average monthly expenditure on dietary supplements is in the range of PLN 20-40 (35.5\% and $29.6 \%$ respectively). According to the data of the Central Statistical Office, in 2019 Poles spent an average of $25.1 \%$ of their income on food and beverages, and the average income per 1 person in the adult population in 2019 was PLN 1819 (29).

Our research shows that students most often chose dietary supplements that contained vitamin $\mathrm{D}$ and / or K and dietary supplements with omega 3 and / or omega 6 and / or omega 9. Earlier literature data indicated that the most popular dietary supplements are preparations with magnesium $(7.56 \%)$ (6). According to our survey results, $8.33 \%$ of students use magnesium supplements. The current epidemiological situation may cause such a large increase in interest in dietary supplements supporting immunity.

Our research also has some limitations. This study was conducted with MUB students - it is a group of people who care about their health, including a properly balanced diet and physical activity. The results of the survey regarding opinions on the marketing of dietary supplements may be different in the case of other populations, for example among students of technical faculties. Further research should also focus on a different selection of the study group: an equal gender distribution and an equal percentage of students from different faculties. The results of the above study also suggest that the composition and labeling of dietary supplements should be more supervised and controlled to ensure the safety and effectiveness of supplementing the diet with dietary supplements.

To conclude, an issue that needs to be developed is a survey among medical university students and health care workers in Poland, regarding their knowledge and opinions about dietary supplements and marketing. These opinions should be taken into account when developing marketing strategies so that advertisements for dietary supplements are not misleading and discouraging, and provide factually correct information, in a condensed form and present dietary supplements with a confirmed composition, which will increase the confidence of patients and health care professionals.

\section{ACKNOWLEDGMENTS}

We would like to give our thanks to everyone who disinterestedly took their time to help us collect the data.

\section{FUND}

This research received no external funding.

\section{CONFLICT OF INTEREST}

The authors report no conflicts of interest.

\section{REFERENCES}

1. Journal of Laws 2006 no. 171 item 1225, the Act of August 25, 2006 on food and nutrition safety (in Polish).

2. Journal of Laws 2018 item 1951, Announcement of the Minister of Health of 17 September 2018 on the publication of the uniform text of the Regulation of the Minister of Health on the composition and labeling of dietary supplements (in Polish).

3. Stoś K., Wierzejska R., Siuba-Strzelińska M.: Dietary supplements - do you need? Instytut Żywności i Żywienia, Warszawa 2019 (in Polish).

4. Matysek-Nawrocka M., Bernat M., Dyczewski B., Chmiel A.: Zeszyty Naukowe WSNS 1, 145 (2016) (in Polish).

5. Brodziak-Dopierała B., Fischer A., Szczelina W., Stojko J.: Biol. Trace Elem. Res. 185, 236 (2018).

6. Suplindex (accessed on 7.01.2021).

7. Kotler P., Keller K.L.: Marketing. Dom Wydawniczy REBIS Sp. z o.o., Poznań 2019 (in Polish).

8. Pacenti G.C., Mancini P.: Marketing of pharmaceutical products. New strategies for the 
healthcare sector. CeDeWu Sp. z o.o., Warszawa 2015 (in Polish).

9. Dietary supplements market in Poland 2019. Market analysis and development forecasts for 2019-2024 (in Polish).

10. Karbownik M.S., Paul E., Nowicka M., Nowicka Z., Kowalczyk R.P., et al.: PLoS One 14, e0218398 (2019).

11. Sigłowa A., Bertrandt B., Conder M., Bertrandt K., Lisiecka A., et al.: Żywn. Nauka Technol. Jakość 4, 236 (2009) (in Polish).

12. Puścion A., Markiewicz-Żukowska R., Karpińska E., Mystkowska K., Borawska M.H.: Bromat. Chem. Toksykol. 45, 912 (2012) (in Polish).

13. Wilczewska B., Cybulski M., Krajewska-Kułak E.: Med. Rodz. 4, 165 (2015) (in Polish).

14. Ratajczak P., Kus K., Poprawa M., Kopciuch D., Paczkowska A., et al.: Pol. Prz. Nauk Zdr. 3, 148 (2019).

15. Morris C.A, Avorn J.: JAMA. 290, 1505 (2003).

16. Krejpcio Z., Staniek H., Chmielewska A.: Probl. Hig. Epidemiol. 94, 622 (2011) (in Polish).

17. Sicińska E., Pietruszka B., Januszko O., Kałuża J.: Nutrients 11, 658 (2019).

18. Vickers A.J., Rees R.W., Robin A.: Clin. Med. (Lond) 32, 426 (1998).
19. Temple N.J., Eley D., Nowrouzi B.: J. Am. Coll. Nutr. 28, 674 (2009).

20. Kowalik M., Mendyk K.M., Klimek M., AntosLatek K., Pagacz K., et al.: Piel. Zdr. Publ. 6, 295 (2016) (in Polish).

21. Bjelica A., Aleksić S., Goločorbin-Kon S., Sazdanić D., Torović L., et al.: J. Altern. Complement. Med. 26, 204 (2020).

22. Ciszek P., Duma P.: Bromat. Chem. Toksykol. 46, 404 (2013).

23. Krejpcio Z., Skwarek K., Hyżyk A.K., Dyba S.: Probl. Hig. Epidemiol. 92, 935 (2011) (in Polish).

24. Michalik M., Pilarczyk B., Mruk H.: Strategic marketing in the pharmaceutical market. Oficyna Ekonomiczna, Kraków 2005 (in Polish).

25. Ethan D., Basch C.H., Samuel L., Quinn C., Dunne S.: J. Community Health 40, 564 (2015).

26. Cardenas D., Fuchs-Tarlovsky V.: Clin. Nutr. ESPEN. 25, 133 (2018).

27. Spiołek K., Kościołek A., Kania J., Hartman M., Pawłowska-Góral K.: Roczn. PZH. 62, 37 (2011).

28. Lobb A.L.: Hawaii J. Med. Public Health 71, 46 (2012).

29. Główny Urząd Statystyczny: Household budgets in 2019, Warszwa 2020 (in Polish). 